Mon. Not. R. Astron. Soc. 000, 1-16 (2015) Printed 10 September $2018 \quad$ (MN LATEX style file v2.2)

\title{
Warps and waves in the stellar discs of the Auriga cosmological simulations
}

\author{
Facundo A. Gómez ${ }^{1 \star}$, Simon D. M. White ${ }^{1}$, Robert J. J. Grand ${ }^{2,3}$, \\ Federico Marinacci ${ }^{4}$, Volker Springel ${ }^{2,3}$ and Rüdiger Pakmor ${ }^{2}$ \\ ${ }^{1}$ Max-Planck-Institut für Astrophysik, Karl-Schwarzschild-Str. 1, D-85748, Garching, Germany \\ ${ }^{2}$ Heidelberger Institut für Theoretische Studien, Schloss-Wolfsbrunnenweg 35, 69118 Heidelberg, Germany \\ ${ }^{3}$ Zentrum für Astronomie der Universitat Heidelberg, Astronomisches Recheninstitut, Monchhofstr. 12-14, 69120 Heidelberg, Germany \\ ${ }^{4}$ Department of Physics, Kavli Institute for Astrophysics and Space Research, MIT, Cambridge, MA 02139, USA
}

Accepted XXX. Received YYY; in original form ZZZ

\begin{abstract}
Recent studies have revealed an oscillating asymmetry in the vertical structure of the Milky Way's disc. Here we analyze 16 high-resolution, fully cosmological simulations of the evolution of individual Milky Way-sized galaxies, carried out with the MHD code AREPO. At redshift zero, about $70 \%$ of our galactic discs show strong vertical patterns, with amplitudes that can exceed $2 \mathrm{kpc}$. Half of these are typical 'integral sign' warps. The rest are oscillations similar to those observed in the Milky Way. Such structures are thus expected to be common. The associated mean vertical motions can be as large as $30 \mathrm{~km} / \mathrm{s}$. Cold disc gas typically follows the vertical patterns seen in the stars. These perturbations have a variety of causes: close encounters with satellites, distant flybys of massive objects, accretion of misaligned cold gas from halo infall or from mergers. Tidally induced vertical patterns can be identified in both young and old stellar populations, whereas those originating from cold gas accretion are seen mainly in the younger populations. Galaxies with regular or at most weakly perturbed discs are usually, but not always, free from recent interactions with massive companions, although we have one case where an equilibrium compact disc reforms after a merger.
\end{abstract}

Key words: Galaxy: disc - Galaxy: evolution - galaxies: evolution - galaxies: interactions - galaxies: kinematics and dynamics - methods: numerical

\section{INTRODUCTION}

Vertical perturbations in the outer regions of galactic discs were first discovered through surveys of $\mathrm{HI} 21 \mathrm{~cm}$ lines (Binney 1992, and references therein). Follow up studies based on large observational samples of stellar discs revealed that many of them also show perturbed vertical structures (e.g. Reshetnikov \& Combes 1998; Ann \& Park 2006; Reshetnikov et al. 2016). The most common morphology is what is known as an S-shaped or 'integral sign' warp, but other kinds of distortion are also present.

Broadly speaking, two major mechanisms produce vertical perturbations of the otherwise flat outer discs of spirals (Sellwood 2013). The first is misaligned accretion of high angular momentum cold gas. Such accretion can result from a close encounter with a gas-rich satellite (e.g. the Magellanic Stream, see Sancisi et al. 2008) or from misaligned infall from the cosmic web or from a cooling hot gas halo (e.g. Jiang \&

* E-mail: fgomez@mpa-garching.mpg.de
Binney 1999; Roškar et al. 2010; Aumer et al. 2013). Such accretion is expected to produce vertical patterns which are most evident in the outer disc gas and in young stars formed from it. The second mechanism is tidal distortion of a preexisting disc by an external perturber, such as a low-mass (perhaps merging) satellite with a small pericentre (e.g. Ostriker \& Binney 1989; Quinn, Hernquist \& Fullagar 1993; Velazquez \& White 1999; Bailin 2003; Kazantzidis et al. 2009; Gómez et al. 2013; D'Onghia et al. 2015) a more massive satellite on a distant fly-by (Vesperini \& Weinberg 2000; Kim et al. 2014; Gómez et al. 2016), or a misaligned outer dark matter halo (e.g. Debattista \& Sellwood 1999; Jiang \& Binney 1999; Shen \& Sellwood 2006).

Several studies have indicated that our own Galactic stellar disc has been vertically perturbed (e.g. LópezCorredoira et al. 2002; Momany et al. 2006). However, the morphology of the vertical structure appears more complex than a traditional S- or U-shaped warp. Rather, an oscillating asymmetry, i.e. a global bending pattern, is suggested, with an amplitude that grows as a function of galactocen- 
tric distance. The first evidence for such a bending pattern was presented by Widrow et al. (2012), who identified a North-South asymmetry in the distribution of a sample of main sequence stars in the Solar Neighborhood $\left(R_{\text {helio }} \lesssim 1\right.$ $\mathrm{kpc}$ and $|Z| \lesssim 2 \mathrm{kpc}$ ) (see also Yanny \& Gardner 2013). $\mathrm{Xu}$ et al. (2015) extended this result by characterizing the vertical distribution of a sample of main sequence stars, obtained from the Sloan Digital Sky Survey (York et al. 2000), as a function of galactocentric distance. They found radially extended oscillatory behaviour in the direction of the Galactic anticentre (see also Price-Whelan et al. 2015). Similar results were obtained by Slater et al. (2014), who used a sample of main sequence turn-off stars from Pan-STARRS1 (Kaiser et al. 2010) to map the anticentre stellar distribution to a heliocentric distance of $\sim 17 \mathrm{kpc}$. This study revealed that the Monoceros Ring (Newberg et al. 2002; Yanny et al. 2003), a large and complex stellar structure in the outer Milky Way disc, exhibits a North-South asymmetry with the southern and northern parts dominating the regions closer and further from the Sun, respectively. More recently, Morganson et al. (2016) extended this analysis by creating threedimensional maps of the Monoceros Ring, concluding that it is well described by two concentric circles alternately seen in the South and the North, suggestive of a wave propagating away from a common origin. It is important to note that the systematics of such vertical oscillations in external galaxies are unknown. Most studies attempting to characterize the vertical structure of discs have focused on edge-on systems in which oscillations are difficult to detect because of projection effects.

The idea that the Monoceros Ring reflects a vertical oscillation of the Galactic disc has been supported by many numerical studies (e.g. Kazantzidis et al. 2008; Younger et al. 2008; Quillen et al. 2009; Purcell et al. 2011; Gómez et al. 2013; Widrow et al. 2014; de la Vega et al. 2015). However, most of these have been based on cosmologically motivated but idealized simulations. Gómez et al. (2016, hereafter G16) presented the first model of a Monoceros-like ring obtained from a fully cosmological simulation of the formation of a Milky Way-mass galaxy. This model reproduces, qualitatively, many of the observed properties of the Monoceros Ring. However, the study included a single simulation, and so could not characterize the expected frequency of such structures in the galaxy population. Here, we address this point by analyzing a suite of 16 high-resolution magnetohydrodynamical simulations of the formation and evolution of a Milky Way-mass galaxy (the Auriga Project, Grand et al. 2016a, hereafter GR16). In Section 2 we briefly introduce the simulations and discuss their main properties. We characterize the present-day vertical structure of their discs in Section 3. In Section 4 we connect this vertical structure with the recent evolutionary history of the individual galaxies. We summarize and discuss our results in Section 5.

\section{SIMULATIONS}

In this paper we analyze a suite of 16 fully cosmological magneto hydrodynamical simulations of the formation of a Milky Way-like galaxy. In the following we summarize the methodology and the main characteristics of these simulations. A more detailed description can be found in GR16.
The simulations were carried out using the $N$-body + moving mesh, magnetohydrodynamics code AREPO (Springel 2010). AREPO solves the gravitational and collisionless dynamics by using a TreePM approach (Springel 2005) and discretizes the MHD equations on a dynamic unstructured Voronoi mesh. These equations are solved with a second order Runge-Kutta integration scheme based on high-accuracy least-square spatial gradient estimators of primitive variables (Pakmor et al. 2016), which is an improvement on the treatment in the original version of AREPO (Springel 2010). A $\Lambda$ CDM cosmology, with parameters $\Omega_{\mathrm{m}}=\Omega_{\mathrm{dm}}+\Omega_{\mathrm{b}}=0.307, \Omega_{\mathrm{b}}=0.048, \Omega_{\Lambda}=0.693$, and Hubble constant $H_{0}=100 h \mathrm{~km} \mathrm{~s}^{-1} \mathrm{Mpc}^{-1}=67.77 \mathrm{~km}$ $\mathrm{s}^{-1} \mathrm{Mpc}^{-1}$, was adopted. The haloes were first identified in a lower resolution simulation of a periodic box of side 100 Mpc. To obtain a sample of relatively isolated MW-size systems, candidate haloes were selected within a narrow mass range centered on $10^{12} \mathrm{M}_{\odot}$ and required to be at least 1.37 $\mathrm{Mpc}$ from any object with more than half their mass. Applying the "zoom-in" technique, the galaxies were re-simulated multiple times at different resolution, increasing the mass resolution in each step by a factor of 8 . After setting up the initial dark matter distribution, gas was added by splitting each original dark matter particle into a dark matter particle-gas cell pair. The masses assigned to each are determined from the cosmological baryon mass fraction, and they are separated by half the mean inter-particle spacing. Their phase-space coordinates are chosen such that the centre of mass and velocity of the pair are those of the original dark matter particle. Here, we focus on the simulations with resolution level 4 , for which the typical mass of a high resolution dark matter particle is $\sim 3 \times 10^{5} \mathrm{M}_{\odot}$, and of an initial gas cell is $\sim 4 \times 10^{4} \mathrm{M}_{\odot}$. The stellar physical gravitational softening length grows with the scale factor up to a maximum of $369 \mathrm{pc}$, after which it is kept constant. The softening length of gas cells scales with the mean radius of the cell, with minimum value equal to the stellar softening length at all times. Note that, in high density regions, gas cells are allowed to become smaller than the gravitational softening length. A resolution study across four resolution levels was presented in GR16. This study shows that many galaxy properties, such as surface density profiles, orbital circularity distributions and disc vertical structures are well converged already at the resolution level used in this work.

The simulations include modeling of the critical physical processes that govern galaxy formation, such as gas cooling/heating, star formation, mass return and metal enrichment from stellar evolution, the growth of supermassive black holes, and feedback from stellar sources and from black hole accretion. The parameters that regulate the efficiency of each process were chosen by comparing the results obtained in simulations of cosmologically representative regions to a wide range of observations of the galaxy population (Vogelsberger et al. 2013; Marinacci, Pakmor \& Springel 2014). Magnetic fields are implemented as described in Pakmor \& Springel (2013). A homogeneous magnetic field is seeded at $z=127$, with strength $10^{-14}$ Gauss oriented (arbitrarily) along one coordinate axis. The choice of direction and strength has little effect on the evolution (Pakmor \& Springel 2013; Marinacci et al. 2015). From now on, we will refer to these simulations as $\mathrm{S} i$, with $i$ enumerating the different initial conditions. In addition, throughout this work 
Warps and waves

Table 1. Table of simulation parameters. The columns are 1) Simulation Id code used in this work 2) Model name in the Auriga Project reference paper (GR16); 3) Virial mass; 4) Virial radius; 5) Stellar mass; 6) Disc stellar mass; 7) Disc radial scale length; 8) Bulge stellar mass; 9) Bulge effective radius; 10) Sersic index of the bulge, and 11) Disc to total mass ratio. See GR16 for definitions

\begin{tabular}{|c|c|c|c|c|c|c|c|c|c|c|}
\hline Id & Run & $M_{\text {vir }}\left(10^{12} \mathrm{M}_{\odot}\right)$ & $R_{\mathrm{vir}}(\mathrm{kpc})$ & $M_{*}\left(10^{10} \mathrm{M}_{\odot}\right)$ & $M_{\mathrm{d}}\left(10^{10} \mathrm{M}_{\odot}\right)$ & $R_{\mathrm{d}}(\mathrm{kpc})$ & $M_{\mathrm{b}}\left(10^{10} \mathrm{M}_{\odot}\right)$ & $R_{\text {eff }}(\mathrm{kpc})$ & $n$ & $D / T$ \\
\hline $\mathrm{S} 1$ & $\mathrm{Au}-5$ & 1.19 & 223.09 & 6.72 & 4.38 & 3.80 & 1.95 & 0.87 & 1.02 & 0.69 \\
\hline $\mathrm{S} 2$ & $\mathrm{Au}-6$ & 1.04 & 213.82 & 4.75 & 3.92 & 4.53 & 0.67 & 1.30 & 1.01 & 0.85 \\
\hline S3 & $\mathrm{Au}-12$ & 1.09 & 217.12 & 6.01 & 4.33 & 4.03 & 1.48 & 1.05 & 1.07 & 0.75 \\
\hline $\mathrm{S} 4$ & $\mathrm{Au}-17$ & 1.03 & 212.77 & 7.61 & 2.67 & 2.82 & 4.14 & 1.11 & 0.71 & 0.39 \\
\hline $\mathrm{S} 5$ & $\mathrm{Au}-18$ & 1.22 & 225.29 & 8.04 & 5.17 & 3.03 & 1.98 & 1.06 & 0.79 & 0.72 \\
\hline $\mathrm{S} 6$ & $\mathrm{Au}-2$ & 1.91 & 261.76 & 7.05 & 4.63 & 5.84 & 1.45 & 1.34 & 0.99 & 0.76 \\
\hline S7 & $\mathrm{Au}-3$ & 1.46 & 239.02 & 7.75 & 6.29 & 7.50 & 2.10 & 1.51 & 1.06 & 0.75 \\
\hline S8 & $\mathrm{Au}-9$ & 1.05 & 214.22 & 6.10 & 3.57 & 3.05 & 2.03 & 0.94 & 0.84 & 0.64 \\
\hline S9 & $\mathrm{Au}-15$ & 1.22 & 225.40 & 3.93 & 3.14 & 4.00 & 0.39 & 0.90 & 1.02 & 0.89 \\
\hline S10 & $\mathrm{Au}-23$ & 1.58 & 245.27 & 9.02 & 6.17 & 4.03 & 2.42 & 1.26 & 0.94 & 0.73 \\
\hline S11 & $\mathrm{Au}-24$ & 1.49 & 240.86 & 6.55 & 3.68 & 5.40 & 2.18 & 0.93 & 0.9 & 0.63 \\
\hline $\mathrm{S} 12$ & $\mathrm{Au}-16$ & 1.50 & 241.48 & 5.41 & 4.77 & 7.84 & 1.00 & 1.56 & 1.18 & 0.83 \\
\hline S13 & $\mathrm{Au}-19$ & 1.21 & 224.57 & 5.32 & 3.88 & 4.31 & 1.02 & 1.02 & 1.13 & 0.79 \\
\hline S14 & $\mathrm{Au}-21$ & 1.45 & 238.64 & 7.72 & 5.86 & 4.93 & 1.48 & 1.36 & 1.29 & 0.80 \\
\hline S15 & $\mathrm{Au}-25$ & 1.22 & 225.30 & 3.14 & 2.59 & 6.30 & 0.76 & 2.44 & 1.69 & 0.77 \\
\hline S16 & $\mathrm{Au}-27$ & 1.75 & 253.81 & 9.61 & 7.21 & 4.21 & 1.70 & 0.92 & 1.00 & 0.81 \\
\hline
\end{tabular}

we will compare our results with those presented in G16, who analyzed an additional simulation performed with the same numerical set-up and mass resolution. We will refer to this simulations as Aq-C4.

Figure 1 shows present-day $V$-band images of stellar surface brightness for the 16 galaxies analysed in this work. Only star particles that at $z=0$ are gravitationally bound to the main galaxy are used in making these images. In all cases, discs are shown face-on. The side length of each panel is indicated in its top left corner, and was chosen so such that the entire disc is visible. As discussed by GR16, these discs have a wide variety of morphologies and extents. Some show multi-arm flocculent spiral structure (e.g. S6 and S12). Others have well defined two-armed spirals (e.g. S15). Several have strong bars (S8 and S10) while others have none (S9 and S13). The main properties of each simulated galaxy are listed in Table 1. The disc/bulge decomposition is made by simultaneously fitting of exponential and Sersic profiles to the stellar surface density profiles. A detailed description of how these parameters were obtained is given in (Grand et al. 2016b).

\section{PRESENT-DAY DISCS VERTICAL STRUCTURE}

Following Gómez et al. (2016), the discs were aligned with the $\mathrm{X}-\mathrm{Y}$ plane by iteratively computing, and aligning with the Z-direction, the total angular momentum of the star particles within cylinders of $7 \mathrm{kpc}$ radius and of decreasing height. The star particles used in this procedure were chosen to be less than 5 Gyr old. To obtain a map of the mass-weighted mean height, $\langle\mathrm{Z}\rangle$, a regular Cartesian mesh of cell size $0.5 \mathrm{kpc}$ aligned with the $\mathrm{X}-\mathrm{Y}$ plane was defined. On each node we centre a $1 \mathrm{kpc}$ radius cylinder of $\pm 5 \mathrm{kpc}$ height and compute the stellar mass-weighted $\langle\mathrm{Z}\rangle$. It is important to notice that these maps are weighted by mass and not by luminosity. Thus, the patterns seen in the vertical distribution do not necessarily follow the younger and brighter stellar populations.
Figure 2 shows the $\langle Z\rangle$ maps for each galaxy. The panel sides match those in Figure 1. Interestingly, the sample of simulated discs exhibits a wide variety of vertical structures. Some are almost flat (e.g. S1, S2, S3, S4 and S5) comprising $\sim 30 \%$ of the sample, while others (e.g. S6, S7, S8, S9, S10 and S11) show strong $m=1$ patterns or warps. The amplitude of the warps can be as large as $\langle\mathrm{Z}\rangle \gtrsim 2 \mathrm{kpc}$ in the outer regions (e.g. S6, S9 and S11). The warp angles $\alpha$ of these five warped galaxies, defined as the angle between the plane of the inner unwarped disc and the line connecting the centre to the outermost tip of the warp, take values $\sim 2.5^{\circ}$, $2.7^{\circ}, 4^{\circ}, 7.4^{\circ}, 2.3^{\circ}$ and $5^{\circ}$, respectively. This is consistent with the range of $\alpha$-values found observationally from optical data (e.g. Reshetnikov \& Combes 1998; Ann \& Park 2006). We find that the warp radius $r_{\mathrm{w}}$, the radius at which the disc bends away from the inner flat disc is, in all cases, located well within the optical radius, $R_{25}$, defined as the radius where $\mu_{\mathrm{B}}=25 \mathrm{mag} / \operatorname{arcsec}^{2}$ (c.f. Pranav \& Jog 2010). We find $r_{\mathrm{w}} \sim 9,7,8.5,5,10,7 \mathrm{kpc}$ for the five galaxies. In all cases, however, we have $r_{w} \gtrsim R_{\mathrm{d}}$, where $R_{\mathrm{d}}$ is the disc radial scale length (see Table 1 ). Warps of this type are $\sim 35 \%$ of of our simulated sample. Note that in all cases these are S-shaped ('integral-sign') warps. Only for S6 we find a clearly asymmetric warp. There is no clear example of a U-shaped warp in our sample.

A third group of galaxies(specifically, S12, S13, S14, S15 and S16, again $\sim 30 \%$ of our sample) show more complicated vertical patterns reminiscent of that found by in Aq-C4 (see G16). In agreement with the empirically derived rules of Briggs (1990), the patterns in many of these galaxies (e.g. Aq-C4, S14 and S16) have a leading spiral morphology that winds with gradually decreasing amplitude into the inner regions. In most cases, these more complex vertical patterns result from the time evolution of an initial integralsign warp. After formation, such warps distort into leading spirals as a result of the torque exerted by the inner, misaligned disc (e.g. Shen \& Sellwood 2006). Note however that a few discs present trailing spiral vertical patterns (e.g. S13 and S15). We will explore the reasons for this in Section 4. 

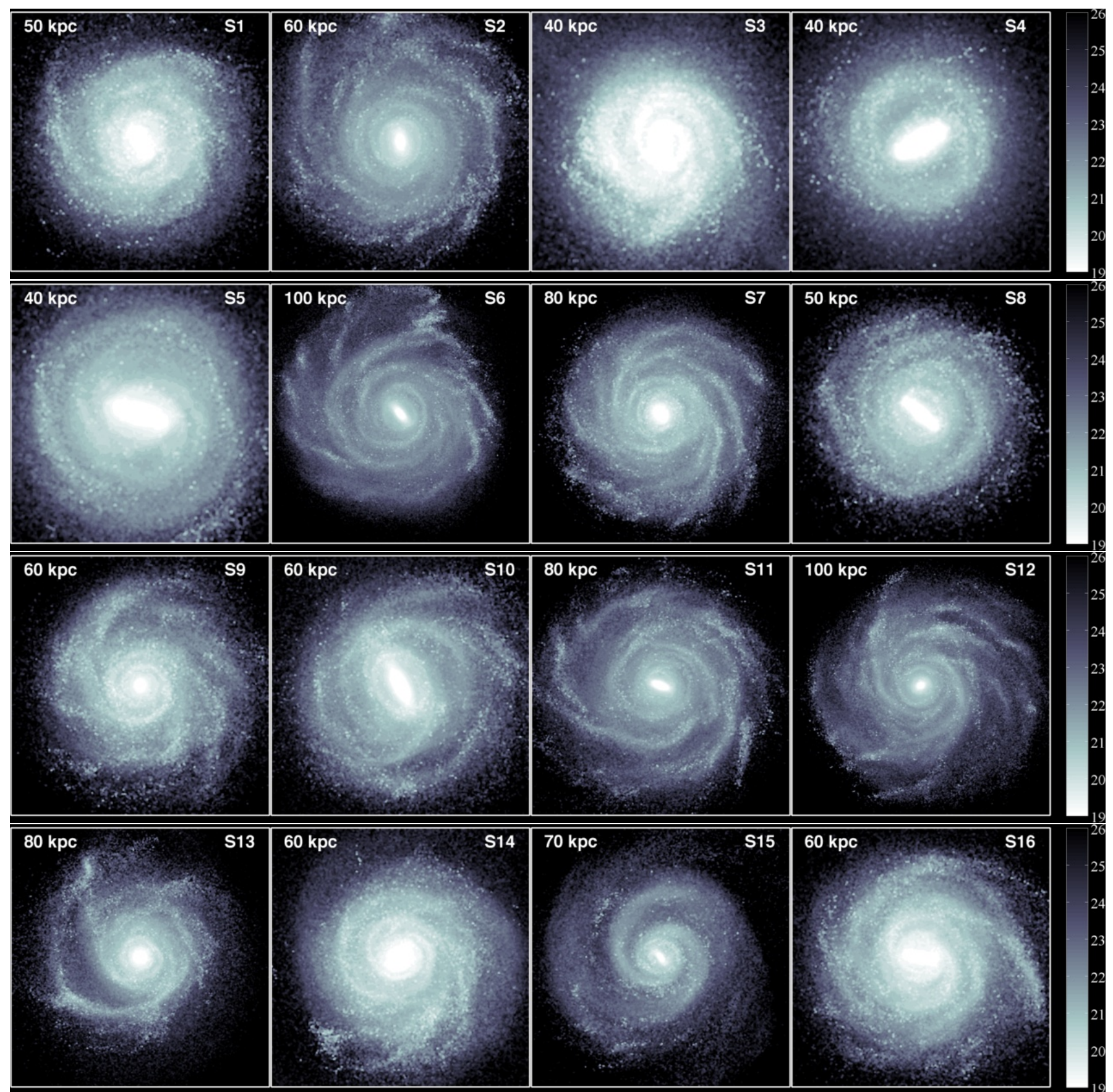

Figure 1. Present-day face-on images of the $V$-band surface brightness, $\mu_{\mathrm{v}}$, of the galaxies analysed in this paper. The side length of each panel is indicated on its top left corner. Only particles that belong to the main host are considered. The colour bar indicates the scale for $\mu_{\mathrm{v}}$ in units of $\mathrm{mag} / \operatorname{arcsec}^{2}$.

The spiral morphology of these patterns is generally not as regular and well defined as that of Aq-C4. Indeed, S13, S14 and S16 could well be classified as S-shaped warps if they were to be observed in certain edge-on orientations. The vertical patterns in this subset of galaxies resembles that observed in the outer MW disc which, as previously described (see Section 1), has an oscillating asymmetry whose amplitude increases with Galactocentric radius (Slater et al. 2014; Price-Whelan et al. 2015; Xu et al. 2015).

Thus, about $70 \%$ of our simulated discs have clearly perturbed vertical structures. The remaining $30 \%$ are either weakly perturbed or featureless. This percentage agrees with observational studies of the frequency of warps, although the definitions used in these studies differ from and are not easily compared with those that we use here (e.g. Ann \& Park 2006, and references therein).

Figure 3 shows mass-weighted mean vertical velocity maps, $\left\langle\mathrm{V}_{\mathrm{z}}\right\rangle$, for each of our discs. These are equivalent to mean (mass-weighted) line-of-sight velocity fields for perfectly face-on galaxies. As expected, galaxies that show 

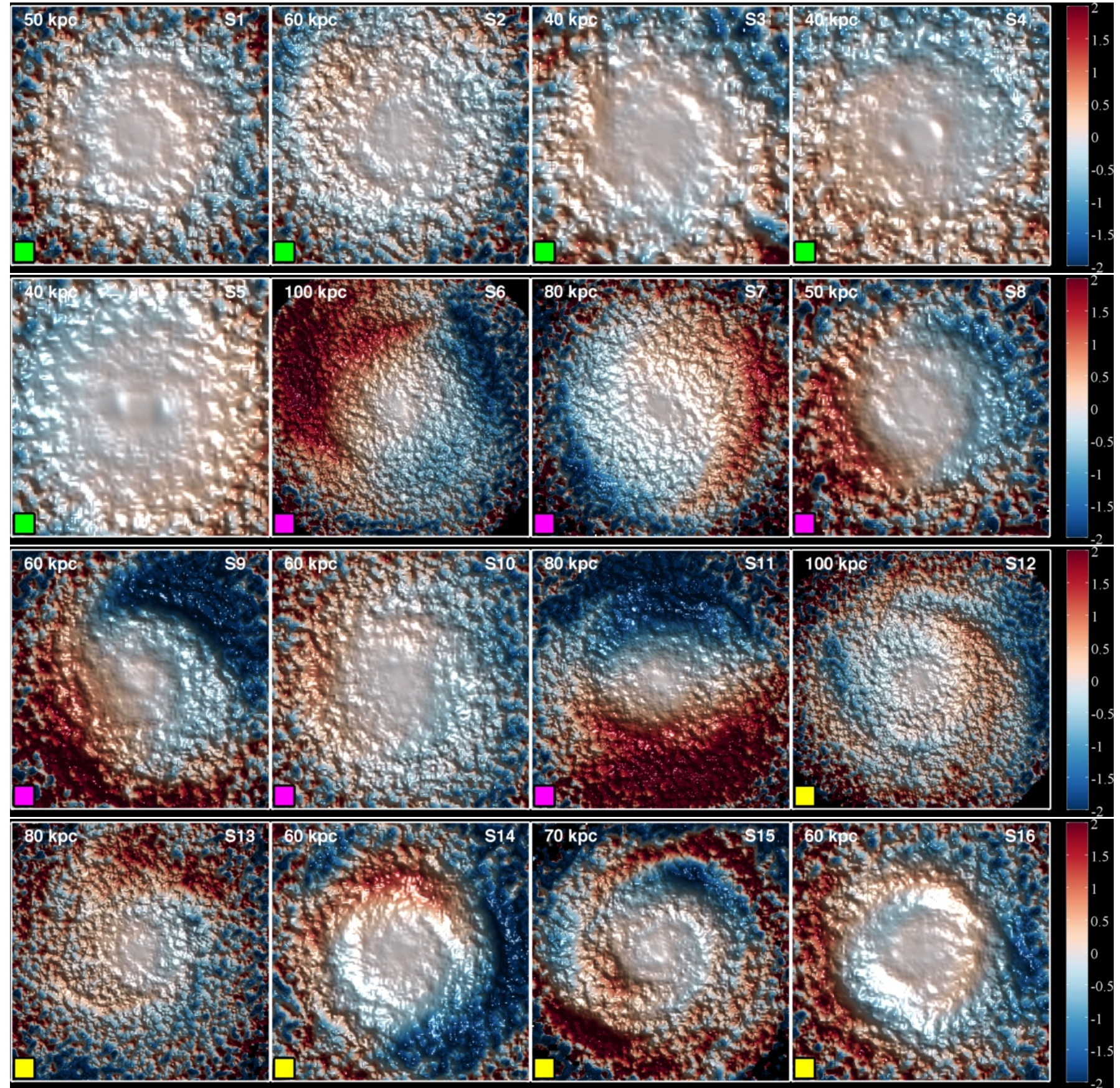

Figure 2. Maps of the simulated stellar disc's mass-weighted mean height, $\langle Z\rangle$, at the present-day. The different map colours and relief indicate different values of $\langle\mathrm{Z}\rangle$ in kpc. The box lengths were chosen to match those used in Figure 1 . The colour coded symbols in the bottom left corner of each panel identify different type of vertical structure; green, magenta and yellow squares indicate weakly perturbed discs, simple warps, and more complex vertical patterns, respectively.

very small perturbations in their $\langle\mathrm{Z}\rangle$ maps also have relatively unperturbed $\left\langle\mathrm{V}_{\mathrm{z}}\right\rangle$ maps. However, large perturbations can be seen in the remaining galaxies, with $\Delta\left\langle\mathrm{V}_{\mathrm{z}}\right\rangle=$ $\left\langle\mathrm{V}_{\mathrm{z}}\right\rangle_{\max }-\left\langle\mathrm{V}_{\mathrm{z}}\right\rangle_{\min }>60 \mathrm{~km} / \mathrm{s}$ in several cases. Perturbations of this kind should be detectable in line-of-sight stellar velocity fields of nearly face-on galaxies obtained from integral field spectroscopy. For example, the Calar Alto Legacy Integral Field Area Survey (CALIFA) provides such velocity fields with uncertainties smaller than $\sim 20 \mathrm{~km} / \mathrm{s}$ (BarreraBallesteros et al. 2014). In addition, such vertical pertur- bations should be visible in HI maps from surveys such as the Local Volume HI Survey (LVHIS). The velocity fields of the galaxy HIPASS J1501-48, presented in Kamphuis et al. (2015), clearly show that complex spiral features can be observed in real face-on galaxies. The HI line-of-sight velocity field of the galaxy UGC09965, recently presented by Martinsson et al. (2016), provides another example. This galaxy, observed for the DiskMass survey (Bershady et al. 2010), also shows clear indications of a complex vertical structure.

Comparison of Figures 2 and 3 reveals the expected 


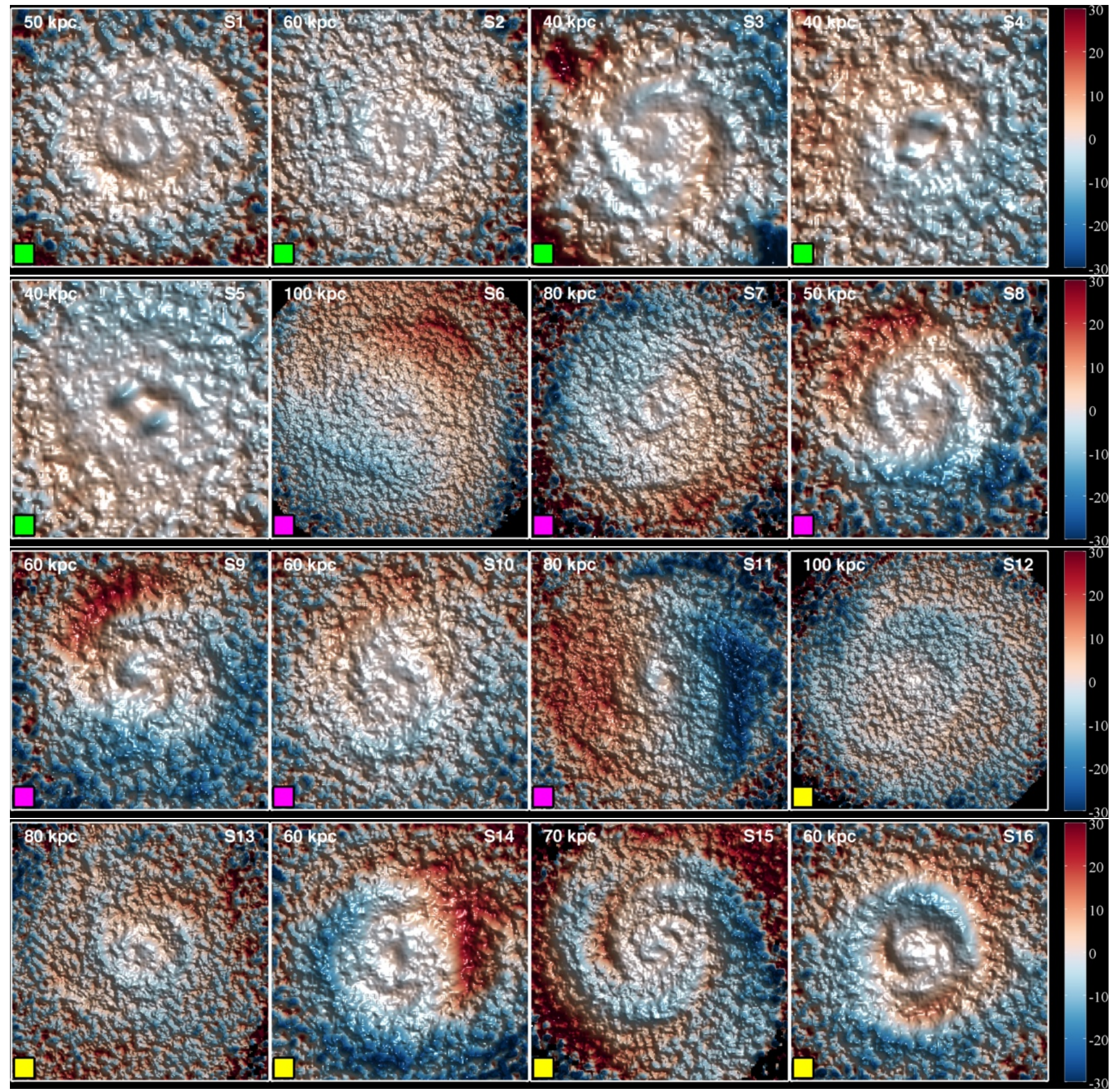

Figure 3. As in Figure 2, but for the mass-weighted mean vertical velocity, $\left\langle\mathrm{V}_{\mathrm{z}}\right\rangle$. The different map colours and the relief indicate different values of $\left\langle\mathrm{V}_{\mathrm{z}}\right\rangle$ in $\mathrm{km} / \mathrm{s}$.

anticorrelation between the absolute values of $\langle\mathrm{Z}\rangle$ and $\left\langle\mathrm{V}_{\mathrm{z}}\right\rangle$ (see Gómez et al. 2013, 2016). This is most evident in warped galaxies, such us S6 and S11, where we see clearly that peaks in $|\langle\mathrm{Z}\rangle|$ correspond to regions of $\left|\left\langle\mathrm{V}_{\mathrm{z}}\right\rangle\right| \sim 0$ $\mathrm{km} / \mathrm{s}$ and vice versa. More wound patterns, such as S14 and S16 show the same behavior. Note that in the approximation of a tightly wound bending wave $Z(R, \phi, t)=$ $\Re\left[Z_{a}(R) \mathrm{e}^{i m\left(\phi-\Omega_{\mathrm{p}} t\right)}\right]$, where $Z_{a}(R)=Z(R) \mathrm{e}^{i \int k d R}$ and $\Omega_{\mathrm{p}}$ the pattern speed (Binney \& Tremaine 2008). Thus, at a given $R, Z \propto \Re\left[\mathrm{e}^{i m\left(\phi-\Omega_{\mathrm{p}} t\right)}\right]$ and $V_{z} \propto \Re\left[i m\left(\Omega-\Omega_{\mathrm{p}}\right) Z\right]$. It follows that line-of-sight velocity fields of face-on discs, such as those presented here, can be used to reconstruct vertical structures even in cases with more complicated $\langle\mathrm{Z}\rangle$ morphologies such as S16.

It is interesting to explore how closely the vertical structure of the cold gas traces the vertical structure of the stellar disc. Here, we focus on the cold star-forming component identified by AREPO which would correspond to the sum of the $\mathrm{HI}$ and $\mathrm{H}_{2}$ components of real galaxies. A detailed characterization of $\mathrm{HI}$ discs is presented in a separate paper (Marinacci et al. 2016). In Figure 4 we show the $\langle\mathrm{Z}\rangle$ maps obtained from this cold star-forming gas in each galaxy. Even 

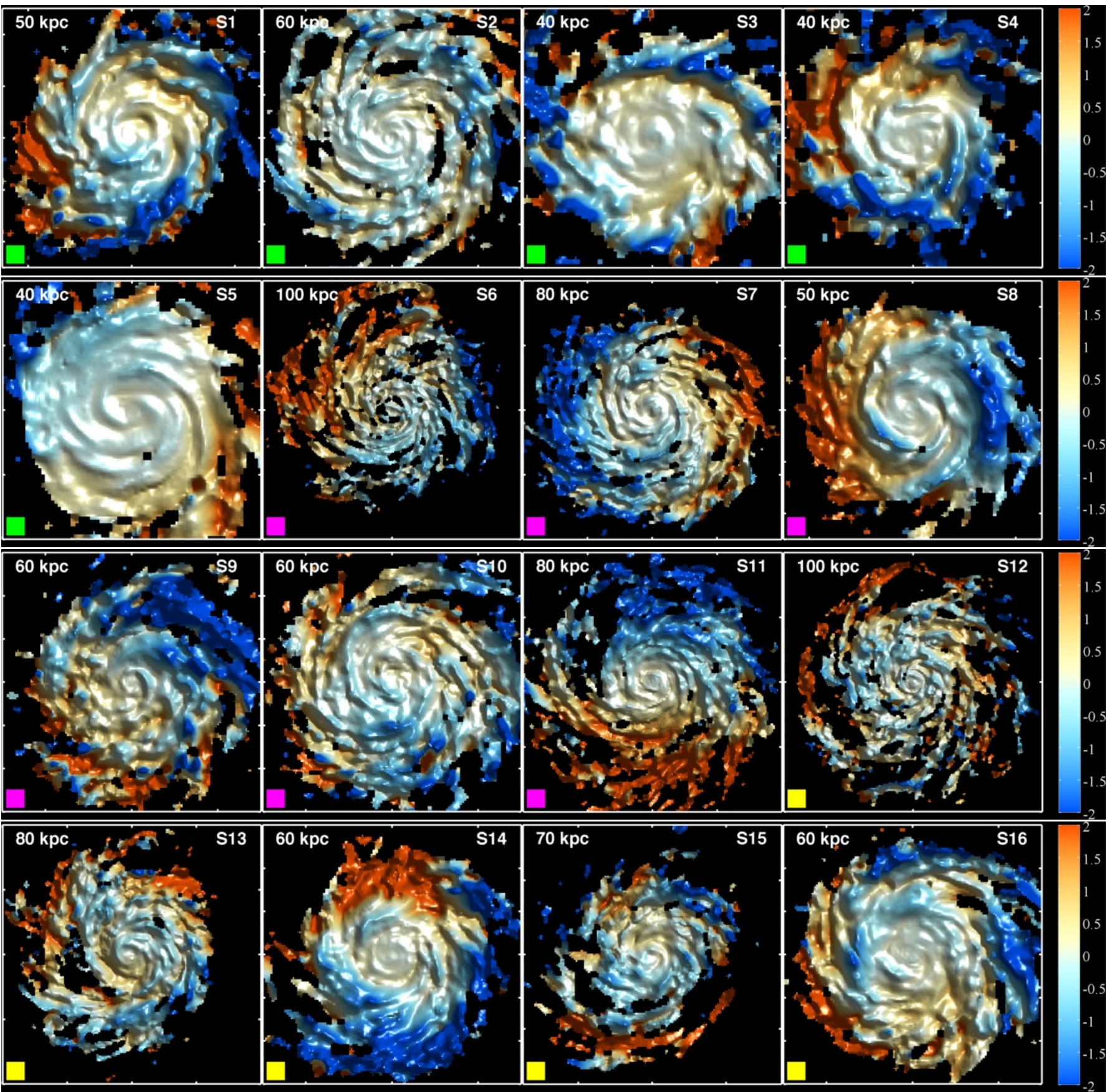

Figure 4. As in Figure 2, but for the cold star-forming gas. The different map colours and the relief indicate different values of $\langle\mathrm{Z}\rangle$ in kpc.

though these maps reveal a significant amount of small scale structure, in general, the gas follows the vertical patterns seen in the stellar discs quite closely. Discs with mildly perturbed $\langle\mathrm{Z}\rangle$ stellar maps, such as S2, S3 and S5, also show featureless or weakly perturbed cold gas maps. In contrast, systems with strongly warped stellar discs (e.g. S6, S7 and S11) show very similar patterns in the cold gas distribution. Note that more complicated vertical patterns, such as those in the stellar discs of S14 and S16, are also reflected in the cold gas distributions. patterns in the stellar and gas discs are quite similar. This suggests either that the patterns are tidally induced in all these cases (see e.g. Cox et al. 1996) or that differences in vertical structure cannot be straightforwardly used to distinguish tidally induced from accretion induced warps. We will explore this further in Section 4. 

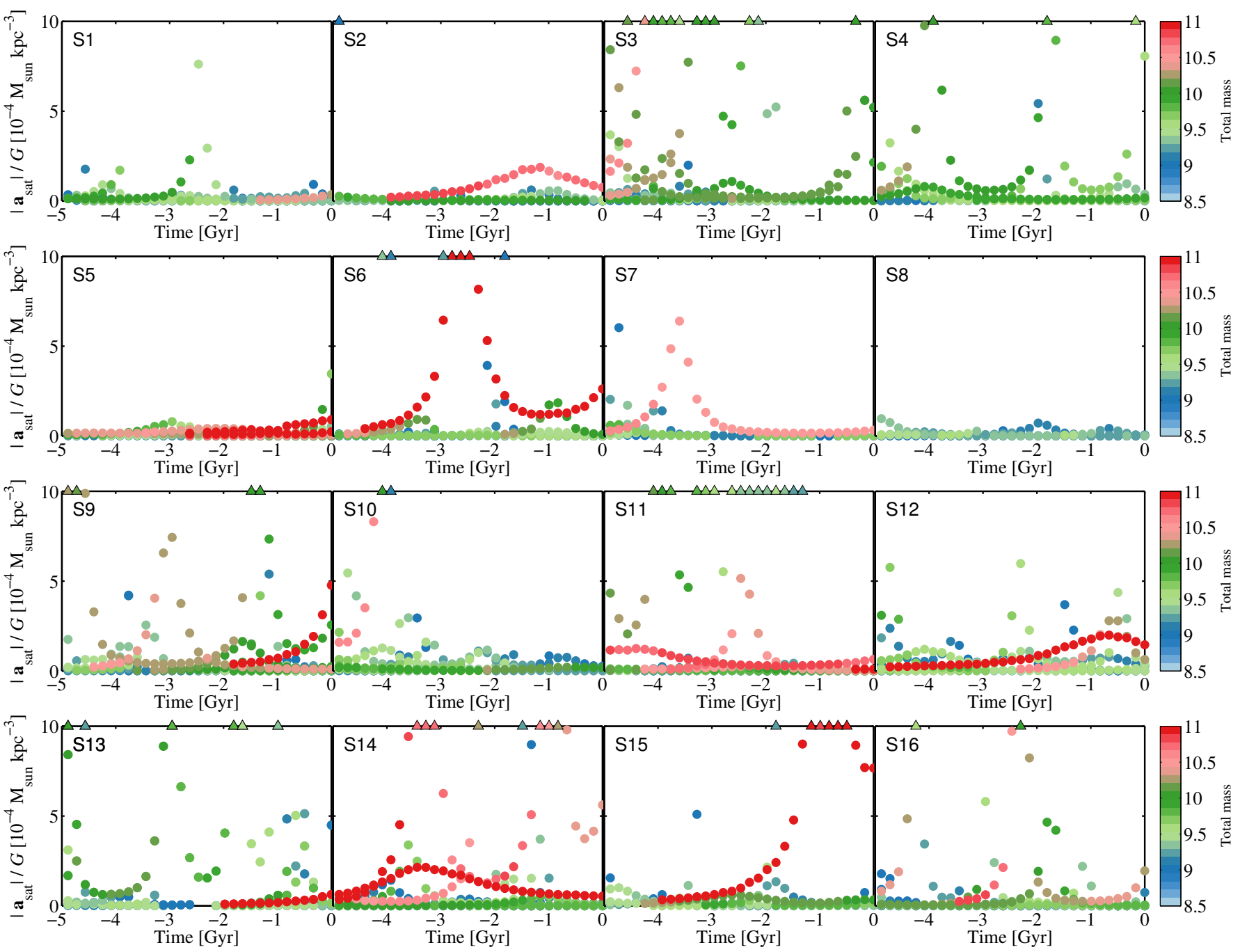

Figure 5. The tidal field exerted on each host by its 20 most massive satellites as a function of time. Points are colour -coded according to the mass of each satellite at the corresponding time. Triangles indicate tidal field values that are above the Y-axis limit.

\section{CONNECTING PRESENT-DAY VERTICAL STRUCTURES WITH GALACTIC HISTORIES}

In this section we will connect the vertical patterns highlighted in section 3 with the recent evolutionary histories of individual galaxies.

As previously discussed, only $\sim 30 \%$ of our discs show unperturbed or weakly perturbed $\langle\mathrm{Z}\rangle$ maps. In the remaining $70 \%$ clear vertical patterns are seen (see Fig. 2). The early work by Hunter \& Toomre (1969) showed that vertical patterns, such as those seen in our simulated discs, can not be long lived stable normal modes. Later attempts to model long lived vertical modes based on the torque exerted by a flattened and misaligned inner dark matter halo (e.g. Sparke \& Casertano 1988) were shown to fail since a misaligned inner halo rapidly aligns with the embedded galactic disc (Binney, Jiang \& Dutta 1998). As a result, such vertical structures are likely to persist only for a few Gyr and to be excited by external perturbations (see Sellwood 2013, for a recent review). Plausible perturbing agents are torques exerted by a misaligned outer dark matter halo, misaligned accretion of cold gas, direct tidal perturbation by orbiting satellites, or torques exerted by the dark matter wakes these satellites produce (e.g. Ostriker \& Binney 1989; Quinn, Hernquist \& Fullagar 1993; Weinberg 1998; Debattista \& Sellwood 1999; Jiang \& Binney 1999; Velazquez \& White 1999; Vesperini \& Weinberg 2000; Shen \& Sellwood 2006; Roškar et al. 2010; DeBuhr, Ma \& White 2012; Kim et al. 2014; Widrow et al. 2014; Gómez et al. 2016). In the following we try to identify the main perturbing agent in each of our simulations.

\subsection{Mildly perturbed discs}

We start by focusing on the subsample of galactic discs that, at the present-day, show relatively unperturbed vertical structure; i.e., S1, S2, S3, S4 and S5. In Figure 5 we show, for each simulated galaxy, the tidal field exerted on the host by its 20 most massive satellites as a function of time, i.e. $\left|\mathbf{a}_{\text {sat }}\right|=G M_{\text {sat }} / R_{\text {sat }}^{3}$. Points are colour coded according to the mass of each satellite at the corresponding time. This figure shows that, in general, the discs in this subset have not interacted closely with any satellite more massive that $M_{\min } \sim 10^{10} \mathrm{M}_{\odot}$ over the last 4 to 5 Gyr. 

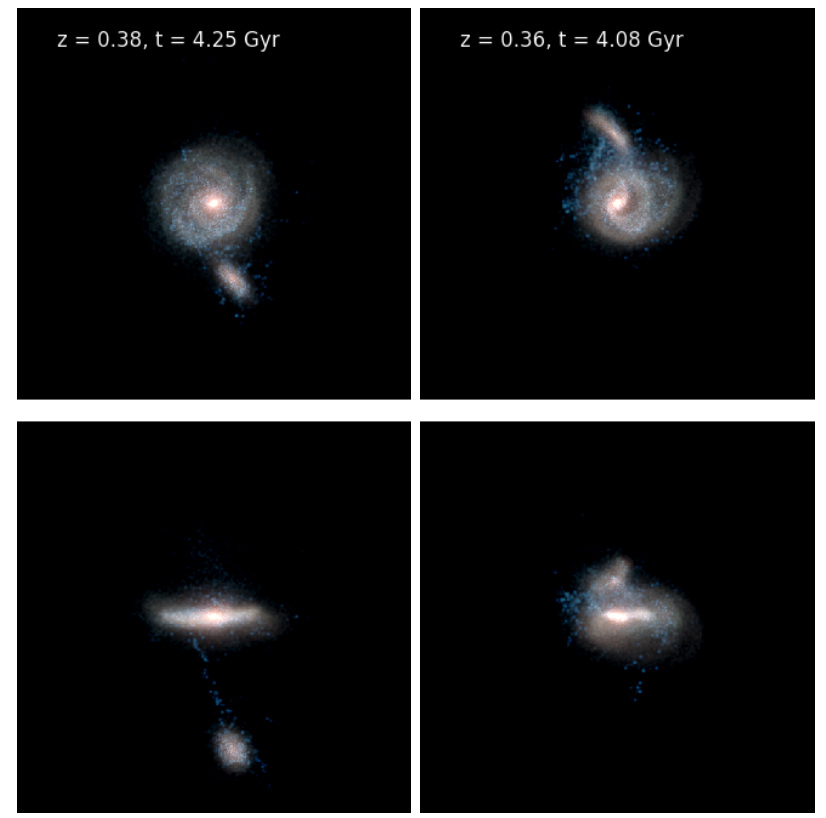

Figure 6. Face-on (top) and edge-on (bottom) projected stellar density for S3 at two different times, as indicated in the top left corner of the top panels. The images were constructed by mapping the K-, B- and U-band luminosities to the red, green and blue channels of a full colour composite image. Younger (older) star particles are therefore represented by bluer (redder) colours. Note that the outer regions of the pre-existing disc are severely perturbed during the head-on encounter with a satellite of $M_{\mathrm{tot}}=10^{10.5} \mathrm{M}_{\odot}$.

It seems that large vertical motions are not easily excited by companions less massive than $M_{\text {min }}$. This agrees with Grand et al. (2016b), who finds that a disc heating in at least a quarter of these simulations appears associated with satellites of masses $>M_{\text {min }}$ but that smaller satellites have negligible effects. Similar results have been recently obtained by Moetazedian \& Just (2015) and D'Onghia et al. (2015).

$\mathrm{S} 3$ is an interesting exception. Unlike the other galaxies in this subset, it experiences a violent interaction $\sim 4 \mathrm{Gyr}$ ago, an almost head-on encounter and subsequent merger with a satellite of mass $M_{\text {tot }} \sim 10^{10.5} \mathrm{M}_{\odot}$. This nearly destroys the outer regions of the disc, induces the formation of a strong bar (see figure 5 of Grand et al. 2016b) and significantly heats the surviving disc. In Figure 6 we can see two simulation snapshots during the merger event. As a result of this interaction, significant inflow of low angular momentum cold gas into the inner disc regions is triggered, substantially boosting the star formation rate at $R \lesssim 10 \mathrm{kpc}$ (Mihos 1999). This can be seen in the top panel of Figure 7 , where we show the time evolution of the disc surface density profile obtained from stars that, at each time, are younger than 1 Gyr. By the present day, S3 has a high surface brightness, compact disc, with $R_{25} \sim 15 \mathrm{kpc}$ (see Figure 1). Small orbital times in this disc combine with a lack of recent perturbations to give a flat system with no significant vertical asymmetries.

In Figure 8 we follow the time evolution of the alignment between each galactic disc and its surrounding dark matter (DM) halo. As in G16, we compute, for every snapshot, the

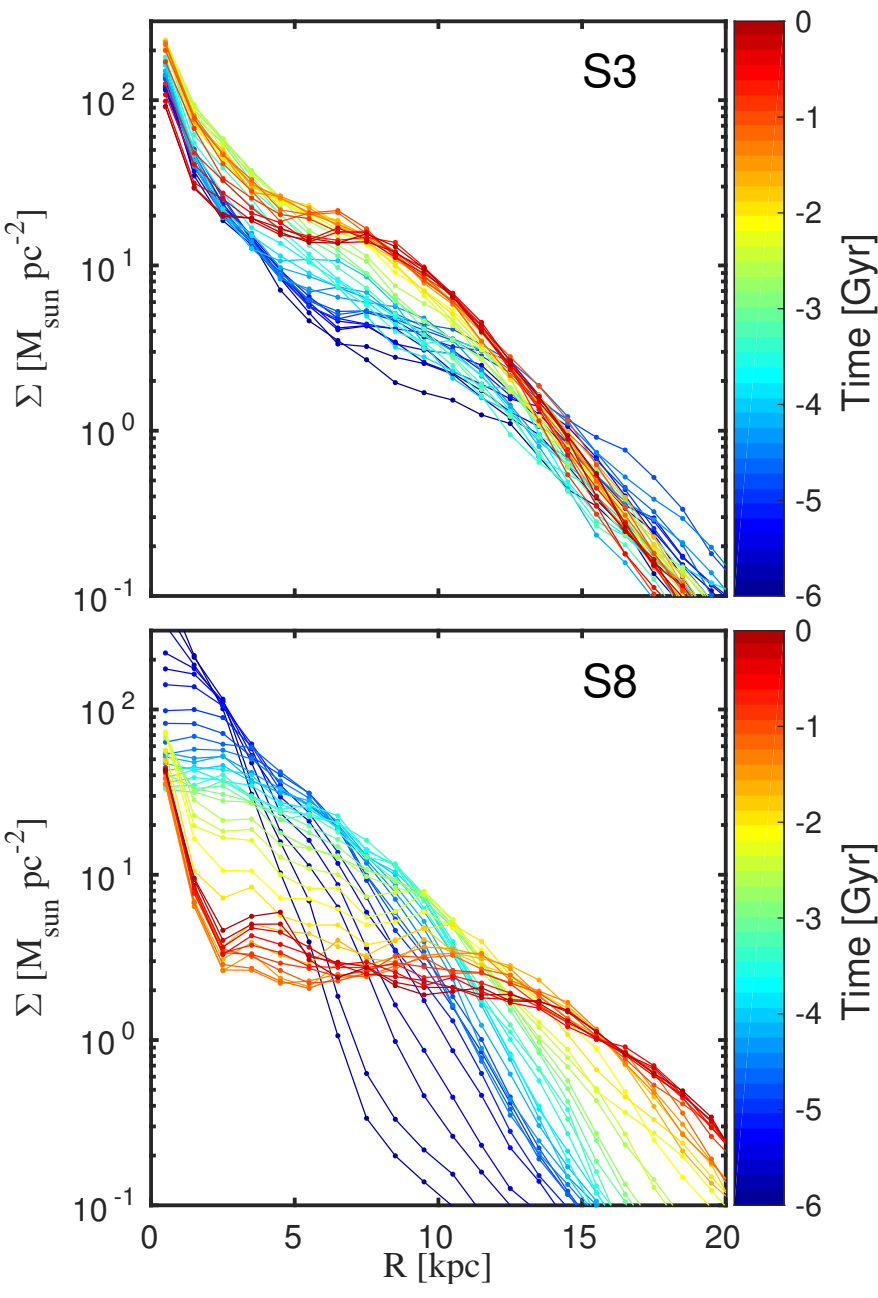

Figure 7. Radial profiles at different times of the mass surface density of star particles younger than 1 Gyr obtained from two different galaxies, as indicated on the top left corner of each panel. Only star particles within $2.5 \mathrm{kpc}$ of the disc mid-plane are considered. The different colours indicate different times.

DM halo inertia tensor,

$$
\mathcal{M}_{\alpha, \beta}=\frac{1}{M} \sum_{i=1}^{N_{p}} m_{i} r_{i, \alpha} r_{i, \beta},
$$

considering only the particles located within various spherical shells. Here $N_{p}$ indicates the total number of particles within the shell, $M$ their total mass, $\boldsymbol{r}_{i}$ and $m_{i}$ the position vector and mass of the $i$ th particle, respectively. $\alpha$ and $\beta$ are tensor indices taking values 1,2 or 3 . We consider four different spherical shells, defined by $0 \mathrm{kpc}<r<10 \mathrm{kpc}, 10 \mathrm{kpc}<$ $r<25 \mathrm{kpc}, 25 \mathrm{kpc}<r<50 \mathrm{kpc}$ and $50 \mathrm{kpc}<r<80 \mathrm{kpc}$. We will refer to them as $\operatorname{dm}(a, b)$, where $a$ and $b$ indicate the lower and upper radial limits of the shell. Once computed, these tensors are diagonalized to obtain the directions of the principal axes.

The different symbols in each panel of Figure 8 show, for each galaxy, the evolution of the angle between the total disc angular momentum and the semi-minor axis of the various DM shells. In general, we find the two innermost DM shells, i.e. $\operatorname{dm}(0,10)$ and $\operatorname{dm}(10,25)$, to be well aligned with the disc at all times, in good agreement with previ- 
ous studies (e.g. Binney, Jiang \& Dutta 1998; Bailin et al. 2005; DeBuhr, Ma \& White 2012; Aumer \& White 2013, G16). At larger radii, some galaxies show increasing dischalo misalignment. Again, S3 is an extreme case in which the two outer shells, $\operatorname{dm}(25,50)$ and $\operatorname{dm}(50,80)$, are severely misaligned at all times shown. Note that the increasing misalignment between the inner and outermost DM shells at $-5<t<-4$ Gyr correlates well with the pericentric passage of the large perturbing satellite previously discussed. Recall that this satellite penetrates deeply into the host DM halo. The two outermost layers continue to be misaligned with the disc angular momentum until the present-day, indicating that the torque exerted by these layers is not strong enough to reorient this disc. Similar correlations between features in the misalignment between disc and outer halo and satellite interactions can also be seen in other galaxies, for example, S1 at $t \sim-2.5 \mathrm{Gyr}$.

In Figure 9 we show the time evolution of the orientation of each inner stellar disc (based on the total angular momentum of disc particles with $r<5 \mathrm{kpc}^{1}$ ) with respect to its orientation at $t=-5 \mathrm{Gyr}$. Excluding S3, all weakly perturbed or unperturbed discs show at most modest reorientation, by angles $\lesssim 20^{\circ}$, over the last 5 Gyr (see e.g. DeBuhr, Ma \& White 2012; Yurin \& Springel 2014). S1 tilts by $\sim 10^{\circ}$ during $-5<t<-3.5 \mathrm{Gyr}$, which correlates well with the misalignment of the DM shell $\operatorname{dm}(10,25)$ during this period. Small jumps in these tilt angles are generally correlated with interactions with intermediate mass satellites, $10^{9.5} \lesssim M_{\text {sat }} \lesssim 10^{10} \mathrm{M}_{\odot}$, as in S1. S3 shows one of the largest disc reorientations in the whole sample which is due almost entirely to its merging with a satellite at $t \approx-4 \mathrm{Gyr}$ with the concomitant near-destruction and reformation of its disc, as already discussed in connection with Figs 6 and 7. A secondary bump can be seen during the last Gyr of evolution, which correlates with a close encounter with $\mathrm{a} \sim 10^{10} \mathrm{M}_{\odot}$ satellite.

In summary, we find that, excluding S3, the galaxies with weak or absent vertical structure have had a quiescent environment over the last 5 Gyr. They show mild disc re-orientations and the inner shells of their DM haloes are always well aligned with the galactic disc. In contrast, S3, suffered a violent interaction at $t \sim-4$ Gyr that nearly destroyed its outer disc and led to the formation of a newer and compact inner disc which effectively resists subsequent perturbations.

\subsection{Strongly perturbed discs}

We now consider the discs that, at the present-day, show significantly perturbed vertical structure. As discussed above, patterns in the $\langle Z\rangle$ maps can be produced by a variety of mechanisms. Here we will not attempt an exhaustive analysis of the history of perturbing torques on a case by case basis, as done by G16 for the Aq-C4 simulation. Rather, we will focus our analysis on general indicators that can help us to identify the nature of the perturbers.

We start by exploring which of these galaxies have recently interacted tidally with a massive companion. Figure 5 shows the time evolution of the tidal field exerted

\footnotetext{
1 Our results are not sensitive to the exact definition here.
}

at system centre by each of the 20 most massive satellites in each system. It demonstrates that most of these galactic discs have had significant interactions with satellites of $M_{\text {sat }} \geq 10^{10.5} \mathrm{M}_{\odot}$ during the last $\sim 4$ Gyr. As shown in G16, even satellites with masses of $\sim 10^{10.5} \mathrm{M}_{\odot}$ and pericentric distances as large as $80 \mathrm{kpc}$ can excite strong vertical patterns in the disc either through their direct tidal interaction or through an associated DM halo wake (see also Vesperini \& Weinberg 2000; Ogiya \& Burkert 2016). Some of these galaxies (in particular, S6, S7, S10, S11 and S15) have interacted primarily with a single massive perturber during this period. The masses at infall of these perturbers are $11^{11.5}, 10^{10.7}, 10^{11}, 10^{10.7}$ and $10^{11.5} \mathrm{M}_{\odot}$, respectively. For example, the massive perturber in S10 had its first pericentric passage at $t=-6 \mathrm{Gyr}$ and undergoes a second close interaction at $t=-4$ Gyr. Other galaxies, such as S9, S14 and S16, have been bombarded by two or more massive satellites. The most massive perturber in these cases has an infall mass of $10^{10.5}, 10^{11.1}$ and $10^{10.9} \mathrm{M}_{\odot}$, respectively. On the other hand S8, S12 and S13 show significantly perturbed $\langle\mathrm{Z}\rangle$ maps but no signs of close tidal interaction with perturbers of $M_{\text {sat }} \geq 10^{10.5}$. This suggests that other mechanisms play a role in these galaxies.

In Figure 8 we explore whether misalignment between different shells of DM could be driving any of these perturbations. In general, galaxies that have interacted mainly with one massive perturber show DM halos that are very well aligned with their galactic disc, even up to distances as large as $80 \mathrm{kpc}$ (see also DeBuhr, Ma \& White 2012). S6, S7, S10 and S11 show only small bumps in the orientation of their outermost DM shells at the time of maximum interaction, indicating that these satellites do not trigger any long-term misalignment. However, we can see from Figure 9 that these discs (and thus the systems as a whole) do change orientation during these interactions. For example, by the present-day, S6 shows a total disc tilt of $\sim 25^{\circ}$ since $t=-5 \mathrm{Gyr}$. The pericentric passage of the perturber coincides with a dip in the time evolution of this tilting at $t \sim-2.9$ Gyr. S11 shows the largest tilt among this subset of discs. Here also, the pericentric passage of the most significant perturber coincides with a bump in the time evolution of the tilt (at $t \sim-2.6 \mathrm{Gyr}$ ). However, the disc shows ongoing re-orientation well before this time, perhaps related to a strong interaction with a different satellite that reached pericenter at $t \sim-5.1 \mathrm{Gyr}$.

In contrast, S15 develops a significant misalignment of its outer halo shell $(\operatorname{dm}(50,80))$ at late times. As discussed earlier, this galaxy undergoes a violent interaction with a $10^{11.5} \mathrm{M}_{\odot}$ companion at $t \sim-0.9 \mathrm{~Gy}$; this correlates well with the main reorientation of the DM shell. Note that, unlike the distant fly-by encounters experienced by S6, S7 and S11 (with pericentric distances of $\sim 80,70$ and $70 \mathrm{kpc}$ respectively) the S15 perturber penetrates deep into the host's inner $40 \mathrm{kpc}$ (see Fig.14 of Grand et al. 2016b). Figure 10 shows two snapshots from this strong interaction. The host disc is tidally perturbed, inducing the formation of two tidal arms. The vertical pattern seen in this simulation traces the trailing structure of these tidal arms.

In general, galaxies that have suffered close encounters, (i.e. S3, S9, S14, S15, and S16) tend to show more misaligned outer DM shells. Features such as bumps and dips in the evolution of the orientation of these shells correlate well with 

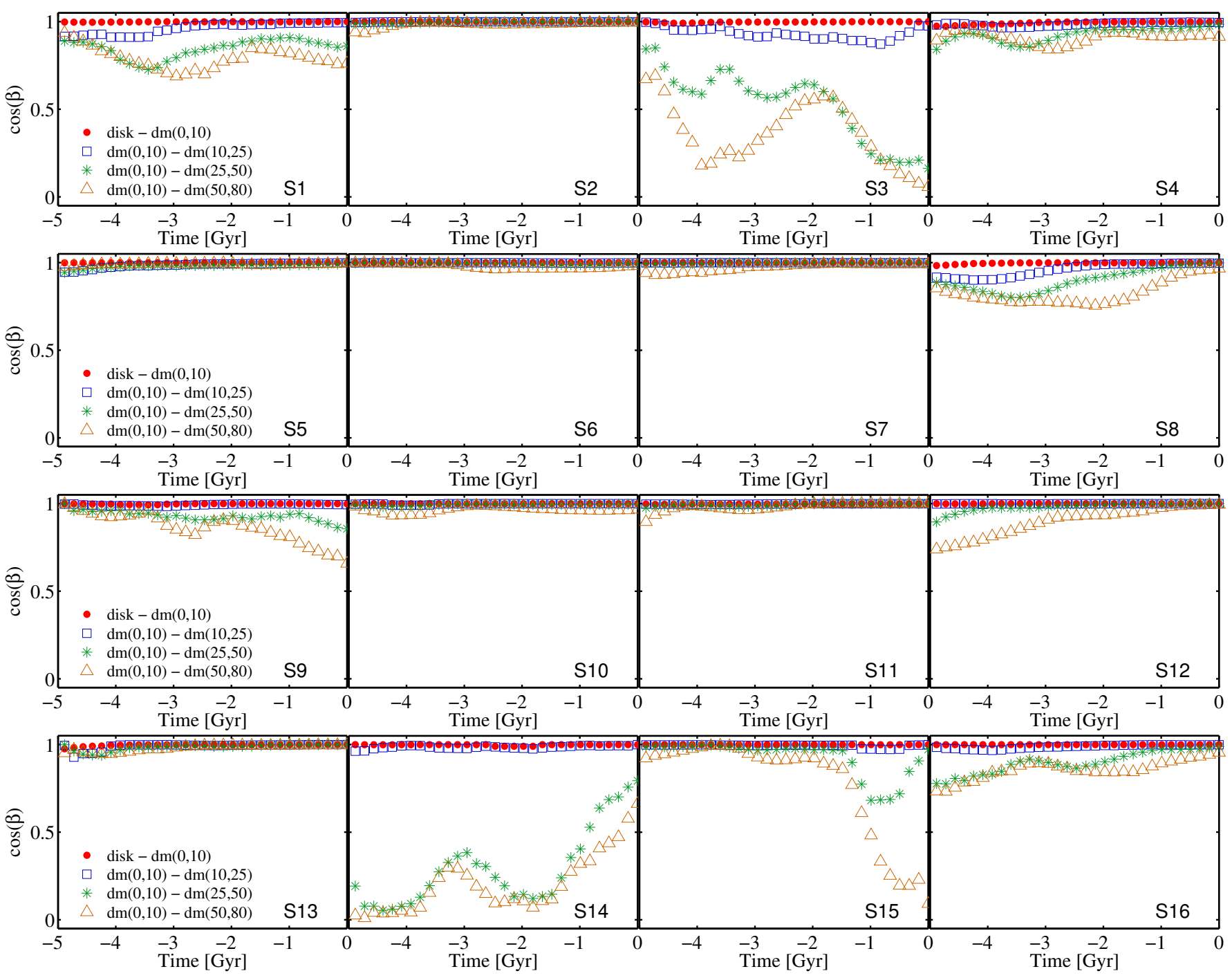

Figure 8. The solid dots indicate the time evolution of the angle between the inner disc and the semi-minor axis of the inner DM halo (see Eq. 1), i.e. $\operatorname{dm}(0-10)$. Open symbols show the time evolution of the angle between the semi-minor axes of different DM shells, as indicated in the legend.

peak satellite interactions. Disc orientation variations also appear to correlate with these interactions in a way which depends, among other things, on the orbital properties of the satellite galaxies (e.g., Velazquez \& White 1999; Villalobos \& Helmi 2008; Kazantzidis et al. 2009).

While a tidal origin is clearly indicated for the vertical patterns in many of our discs, other cases seem to require another explanation. A specific example is $\mathrm{S} 8$, which shows a well-defined S-shaped warp (see Fig. 2). As can be seen from Figure 5, this galaxy has not interacted closely with any satellite for at least the last 5 Gyr. Nevertheless, Figure 8 reveals a significant misalignment of its DM halo shells, especially at $t \lesssim-2$ Gyr. Even the inner $\operatorname{dm}(10,25)$ DM shell is slightly misaligned with respect to the disc plane. This misalignment correlates well with the tilting of the disc over this period (Fig. 9), suggesting that torques exerted by the DM halo are driving the evolution. Over the last 2 Gyr of evolution, however, the DM halo within $50 \mathrm{kpc}$ aligns well with the disc plane and the overall tilting of the system slows. Despite this, we will see below that the warp in S8 grows significantly over the final 0.5 Gyr. DM torques seem unlikely to be the primary driver of this late evolution.

A mechanism which can excite vertical patterns in discs in the absence of external tidal perturbations is the accretion of misaligned cold gas (see e.g. Sancisi et al. 2008; Aumer et al. 2013). For example, Roškar et al. (2010, hereafter R10) analyse a simulation in which a warp forms as a result of misalignment between the inner stellar disc and a surrounding hot gas halo. As discussed in G16, in this situation, cold gas falling into the system is strongly affected by the hot gas halo, and by the time it reaches the disc, its angular momentum aligns with that of the hot gas, rather than that of the disc. A misaligned outer disc then forms from the newly accreted material. A characteristic of this mechanism is that it does not produce significant warping of the pre-existing disc, and the outer stellar warp is heavily dominated by newly formed stars. This contrast with tidally induced warps which typically contain stars of all ages (see e.g. G16).

This mechanism has recently been suggested for the 

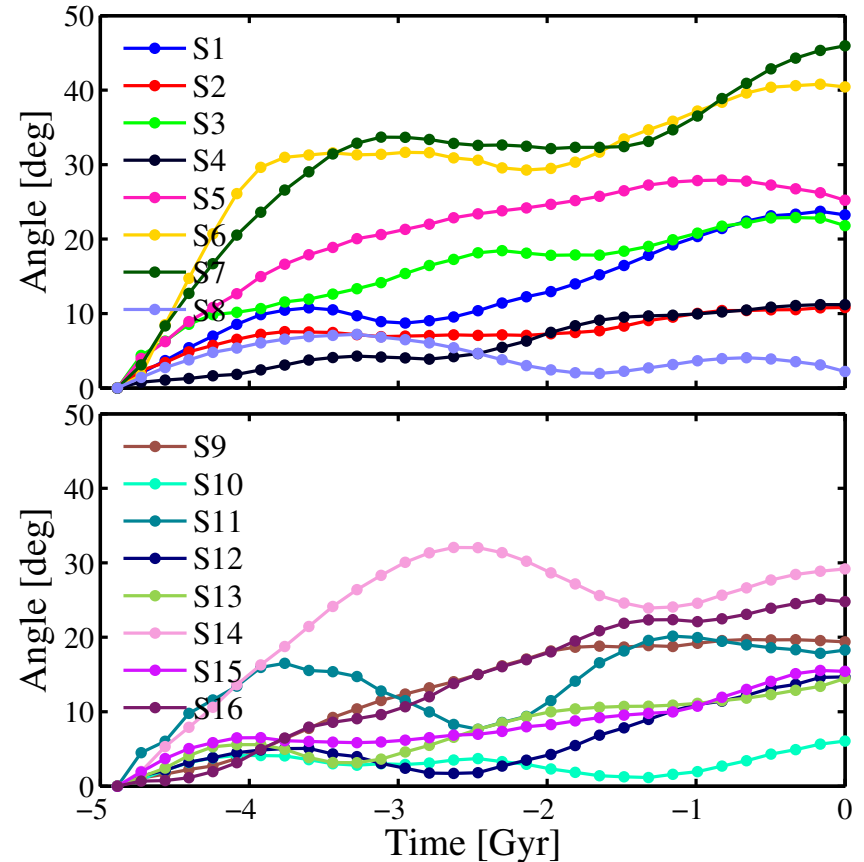

Figure 9. Time evolution of the inner disc orientation with respect to its orientation at $t=-5$ Gyr. Different colours correspond to different simulated galaxies, as indicated in the legend.

formation of the stellar warp in NGC 4565. Using observation from the Hubble Space Telescope, Radburn-Smith et al. (2014) showed that stars older than 1 Gyr lie in a symmetrical distribution around the disc plane. In contrast, a clear and strong warp can be seen in stellar populations younger than $600 \mathrm{Myr}$ and correlates well with the observed HI warp.

In Figure 11 we show $\langle Z\rangle$ maps obtained from stars with ages between 4 and 6 Gyr in four discs that have undergone strong satellite interactions during the last $\sim 4 \mathrm{Gyr}$; namely S6, S7, S14 and S11. In all cases, very strong vertical patterns can be seen in these older stellar populations. The shape and amplitude of these patterns are very similar to those obtained when all stars are included (see Fig. 2), in good agreement with expectations for tidally excited features. Note that the vertical patterns in these old populations also correlate well with those seen in the distribution of cold star-forming gas. This indicate that patterns in the gas and stellar components of a disc can remain coincident for more than 1 Gyr. Similar results are found for halos S9, S15 and S16.

$\langle$ Z $\rangle$ maps for old star particles (ages between 4 and 6 Gyr) in the remaining simulations with strong, present-day vertical patterns are shown in the top panels of Figure 12. With the exception of S10, these galaxies have not interacted with any satellite more massive than $10^{10.5} \mathrm{M}_{\odot}$ over the last $\sim 5$ Gyr. In contrast to the galaxies just discussed, they show at most weak signatures of vertical structure in their old stellar populations. Some structure is seen in S12, but it is much weaker and less complex than when all stars are considered (compare with Fig. 2). The bottom panels of Figure 12 show $\langle Z\rangle$ maps for these same galaxies constructed using only stars younger than 2 Gyr. These panels show much stronger vertical patterns which correlate well with those found for the full stellar populations (Fig. 2)
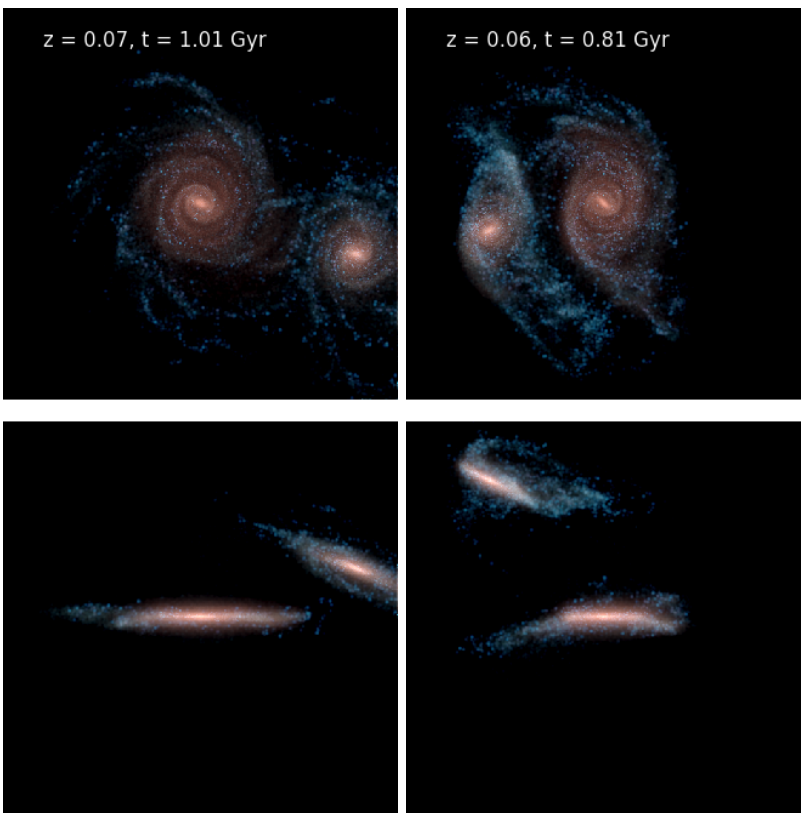

Figure 10. As in Figure 6, but for S15. At $t \sim-1$ Gyr this galaxy has a close encounter with a massive companion with an infall mass of $\sim 10^{11.5} \mathrm{M} \odot$

showing that present-day vertical structure in these discs is dominated by young stars.

S8 is a particularly clear example of a warp dominated by newly formed stars. The bottom panel of Figure 7 shows surface density profiles for this disc made using star particles that, at each time, are younger than 1 Gyr. Formation is evidently inside-out, as expected for a disc that grows continuously by smooth accretion of gas (see, for example, Naab \& Ostriker 2006). At $t \sim-5$ Gyr, star formation is centrally concentrated. However, as cold gas is consumed in the inner regions, a central depression develops in the star formation rate (SFR). At later times, stars are formed at progressively larger galactocentric distances from newly accreted, high angular momentum gas, producing a dominant population of young stars at large radii where the warp is seen. This is the opposite of the behaviour discussed above for S3 (top panel of Fig. 7) where a merger with a massive companion at $t \sim-4$ Gyr triggered an inflow of cold gas to the inner regions, boosting star formation there.

In Figure 13 we show $\langle\mathrm{Z}\rangle$ maps of the S8 disc at two recent times; $t=-0.33$ and -0.66 Gyr (left and right columns, respectively). The bottom and top rows are based on stars younger than $2 \mathrm{Gyr}$, and with ages between 4 and 6 Gyr, respectively. While the warp in the younger component becomes stronger with time, the older component remains quite flat.

S12 is another interesting case. Comparing Figures 2 and 12 we see that the $\langle\mathrm{Z}\rangle$ map for the total stellar mass combines the vertical patterns found in the older and the younger populations, The former is apparently due to tidal effects, while the latter results from accretion of misaligned gas. Note that the $\langle\mathrm{Z}\rangle$ maps found for the two subsets of stars are very different,

S13 and S10 are rather similar to S3. Each merges with a massive companion (at $t=-5$ and $4.2 \mathrm{Gyr}$, respectively). 
The disc of S10 (which has $R_{25} \sim 17 \mathrm{kpc}$ at $t \sim-5 \mathrm{Gyr}$ ) is strongly perturbed by the merger and forms a strong bar. However, unlike S3, it continues to accrete high angular momentum gas and regrows an extended disc by the presentday. This is visible in Figure 2 of Grand et al. (2016b), where stellar surface density is plotted as a function of time. The warps in the young stellar populations of this galaxy are again due to misaligned gas accretion. In contrast, the preexisting disc of S13 is completely destroyed by its gas-rich merger, but a new thin disc quickly forms thereafter due to the re-accretion of misaligned gas (see Figure 14). Unlike S8, where the accreted gas is new material arriving from large radius, the newly formed disc in $\mathrm{S} 13$ is made from gas previously associated with the merging galaxies (Schweizer \& Seitzer 1992; Springel \& Hernquist 2005). This misaligned accreted gas, which shares the sense of rotation of the newly formed $\operatorname{disc}^{2}$, develops a vertical pattern with a trailing spiral morphology.

In summary, we find that vertical structure in our discs is generated by a variety of mechanisms. Close encounters with large companions result in tidally induced patterns that are present in both young and old stellar populations. Mergers with massive and gas-rich satellites can severely perturb or even destroy pre-existing discs, but subsequent accretion of misaligned gas can produce new discs with vertical features that are significantly stronger in the youngest stellar populations. We have also found warps in galaxies that have not interacted with any massive satellite for at least 5 Gyr. Such patterns are generally the result of misaligned gas accretion either from new infall or from residual gas from an earlier gas-rich merger.

\section{SUMMARY AND CONCLUSIONS}

In this study we have characterized the vertical structure of galactic stellar discs in a suite of fully cosmological simulations of the formation and evolution of individual Milky Way-like galaxies. Based on the state-of-the-art, $N$-body magnetohydrodynamical code AREPO, the galactic discs in these simulations show a wide range in morphology and extent at $z=0$, including strong bars, flocculent multi-arm spirals, grand design spirals, and compact high surface brightness discs. The simulated Milky Way sized systems were selected to be located at least $1.37 \mathrm{Mpc}$ from any object more than half of their mass. In addition, we have focused our analysis on a subset of haloes that exhibit clear stellar discs at the present-day, in order to characterize their vertical structure.

For each galactic disc, we have constructed maps of the mass-weighted mean height, $\langle\mathrm{Z}\rangle$, for both the stellar and the cold gas components. Approximately $30 \%$ show little or no vertical structure, but the remainder show clear vertical patterns, with amplitudes that can be as large as $2 \mathrm{kpc}$. This fraction of $70 \%^{3}$ agrees with observational studies of large samples of edge-on spirals (e.g. Ann \& Park 2006). We identify four mechanisms producing such structure: close satellite encounters, distant flybys of massive companions, ac-

2 See Aumer et al. (2013) for the formation of counter-rotating discs on top of pre-existing discs due to misaligned infall of gas.

3 Including the simulation Aq-C4 studied in G16. cretion of misaligned cold gas, and re-accretion of cold gas from the progenitors of a gas-rich major merger. Half of our vertically structured discs show "S-shape" or "integral sign" warps, with a distribution of warp angle, $\alpha$, centered around $\langle\alpha\rangle=4^{\circ} \pm 2^{\circ}$, which agrees well with observational estimates (e.g. Reshetnikov \& Combes 1998; Ann \& Park 2006). In all cases, we find the warp to begin well within $R_{25}$ but outside one disc scale-length (c.f. Pranav \& Jog 2010). There is no clear example of a U-shaped warp in our sample. The other vertically structured discs show more complex patterns, mostly with a spiral morphology that winds into the inner disc and is reminiscent of the structure seen in the Milky Way (Slater et al. 2014; Price-Whelan et al. 2015; Xu et al. 2015; Morganson et al. 2016). Including the simulation Aq-C4 from G16, 35\% of our simulated discs show an oscillating vertical asymmetry, suggesting that such behaviour should be quite common. A resolution study performed on two galactic discs shows that the amplitude and morphology of the vertical perturbations are initially well converged. Nonetheless, the damping time-scales of the patterns can differ at different resolution levels. It is also important to highglight that, overall, our final simulated stellar discs are thicker than observed ( $h_{\mathrm{z}} \sim 1 \mathrm{kpc}^{4}$, see Grand et al. 2016a). Thus, their vertical rigidity is significantly lower than that of the Milky Way disc.

We have also explored how closely vertical structure in the cold star forming gas traces that in disc stars. In general, we find that gas and stars follow the same overall pattern, albeit with significant differences on small scales. This is true even for discs with complex oscillatory patterns, and appears independent of the mechanism driving the vertical perturbations. Our results also indicate that the vertical structure of the cold gas and the stellar discs can remain coincident for more than 1 Gyr.

Maps of the stellar mass-weighted mean vertical velocity $\left\langle\mathrm{V}_{\mathrm{z}}\right\rangle$ confirm the oscillatory behavior of these features. Galaxies with strongly perturbed $\langle\mathrm{Z}\rangle$ maps also have significantly perturbed $\left\langle\mathrm{V}_{\mathrm{z}}\right\rangle$ maps, and a clear anticorrelation between the absolute values of $\langle\mathrm{Z}\rangle$ and $\left\langle\mathrm{V}_{\mathrm{z}}\right\rangle$ is visible in all cases (see also Gómez et al. 2013, 2016). Thus, $\left\langle\mathrm{V}_{\mathrm{z}}\right\rangle$ maps can be used to reconstruct vertical structure in a disc, even for complicated morphologies. The vertical velocity perturbations can be as large as $\Delta\left\langle\mathrm{V}_{\mathrm{z}}\right\rangle=\left\langle\mathrm{V}_{\mathrm{z}}\right\rangle_{\max }-\left\langle\mathrm{V}_{\mathrm{z}}\right\rangle_{\min }>60$ $\mathrm{km} / \mathrm{s}$. Such perturbations should be easily detectable in nearly face-on galaxies either from line-of-sight stellar velocity fields obtained by integral field spectroscopy, or from cold gas velocity fields obtained by radio interferometry. Such observations thus provide a direct way to assess the frequency with which oscillating vertical patterns arise in real late-type galaxies.

We have explored the connection between recent assembly history and present-day vertical structure for our suite of Milky Way-like simulations. We find that most (but not all) discs with little or no vertical structure have had no interaction with a satellite more massive than $M_{\min } \sim 10^{10} \mathrm{M}_{\odot}$ over the last 4 to 5 Gyr. It appears that strong, long-lasting vertical patterns are rarely excited by satellites of mass $\lesssim M_{\text {min }}$. Similar results based on idealized but cosmolog-

4 Note that the young stellar discs show a much thiner vertical distribution 


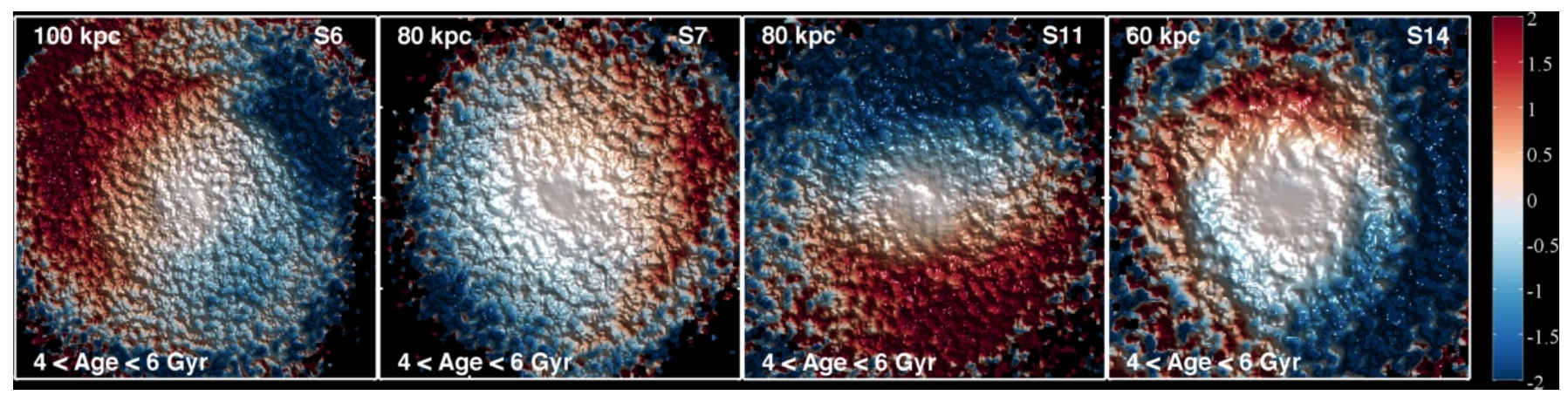

Figure 11. Present-day maps of mass-weighted mean height, $\langle\mathrm{Z}\rangle$, for four stellar discs, based purely on stars with ages between 4 and 6 Gyr. The colour-code and the relief show the value of $\langle\mathrm{Z}\rangle$ in $\mathrm{kpc}$ as indicated by the colour bar. Each of the galaxies in this figure has undergone a strong tidal interactions with a massive satellite during the previous 4 Gyr.

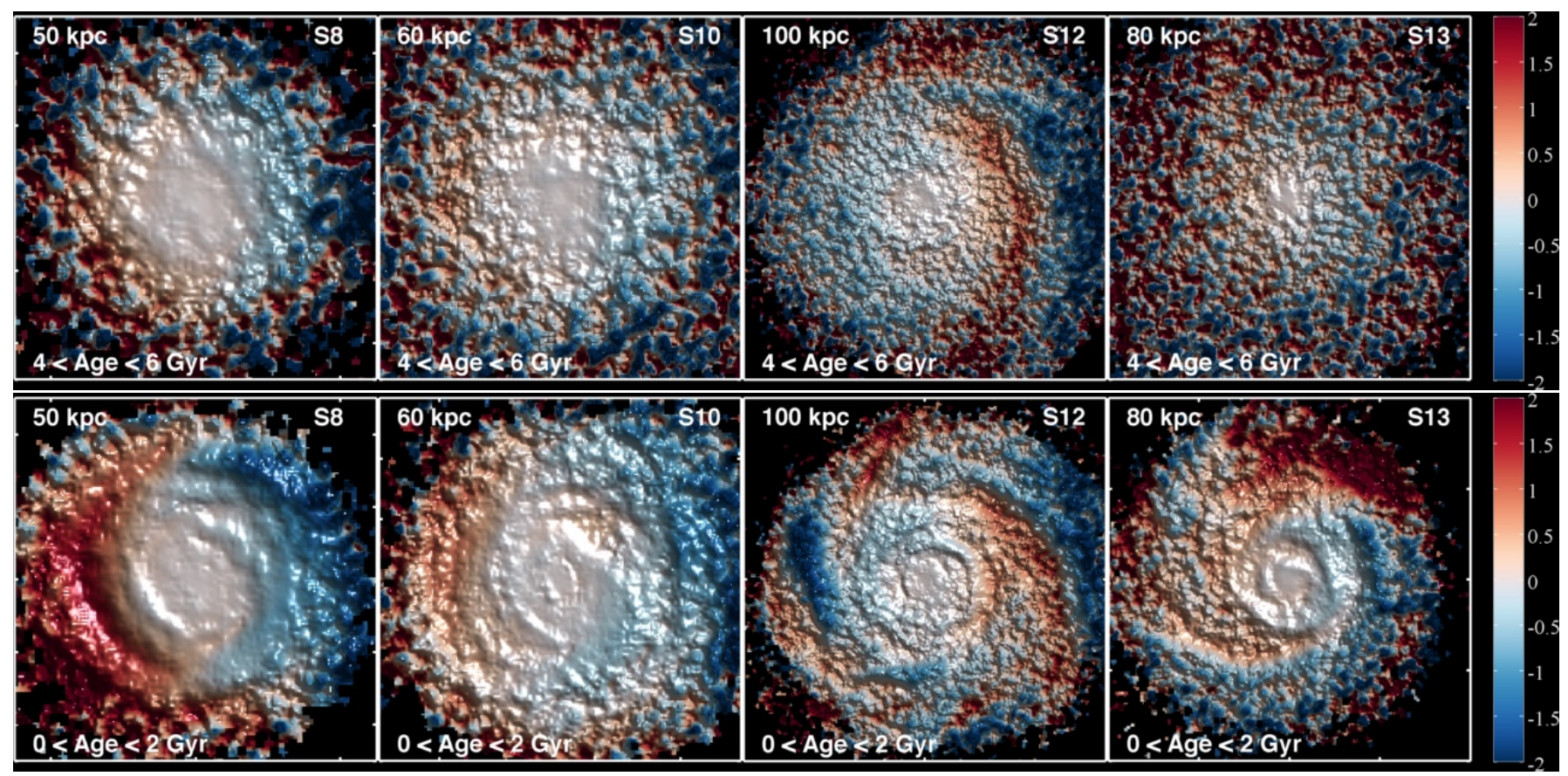

Figure 12. Top panels: As in Figure 11, but for four galaxies that have not interacted strongly with a massive companions during the last 4 Gyr. Bottom panels: Present-day $\langle Z\rangle$ maps for these same four galaxies, but now based on stars with ages between 0 and 2 Gyr.

ically motivated simulations have recently been reported by D'Onghia et al. (2015). This is also in agreement with Grand et al. (2016b) who finds that such perturbations are a significant source of disc heating in at least a quarter of our simulations, and that satellites less massive than $M_{\text {min }}$ play a negligible role in disc heating (see also Moetazedian \& Just 2015). Note, however, that $M_{\text {min }}$ should not be interpreted as a rigid mass threshold; whether or not a largescale bending pattern is excited depends not only on the perturber mass but also on its pericentric distance and velocity (Widrow et al. 2014; D’Onghia et al. 2015).

We note that a present-day vertically relaxed disc does not necessarily imply a quiescent evolutionary history over the last $\sim 5$ Gyr. In particular, our sample includes one example (S3) of a galaxy that merged with a $10^{10.5} \mathrm{M}_{\odot}$ satellite $\sim 4$ Gyr ago. This merger destroyed the outer regions of the pre-existing disc, and triggered the inflow of residual low angular momentum gas to the inner disc, substantially boosting the star formation rate within $10 \mathrm{kpc}$. As a result, the present-day disc is compact and has high surface density, allowing it to come to equilibrium well before $z=0$.

Most galaxies with strong, present-day vertical patterns have interacted at least once with a satellite more massive than $10^{10.5} \mathrm{M}_{\odot}$ during the last 4 to 5 Gyr. As shown by previous studies, low-velocity and relatively distant encounters with satellites with masses as small as $10^{10.5} \mathrm{M}_{\odot}$ can significantly perturb a galactic disc thanks both to direct tidal interaction and to the strong response such flybys can excite in the galaxy's DM halo (e.g. Vesperini \& Weinberg 2000; Gómez et al. 2016).

We have examined whether a misalignment between discs and their DM haloes could be the main driver behind any of the warps in our simulations. In agreement with previous studies (e.g. Binney, Jiang \& Dutta 1998; Bailin et al. 2005; DeBuhr, Ma \& White 2012; Aumer et al. 2013; Gómez et al. 2016), we find that, in almost all cases, disc angular 


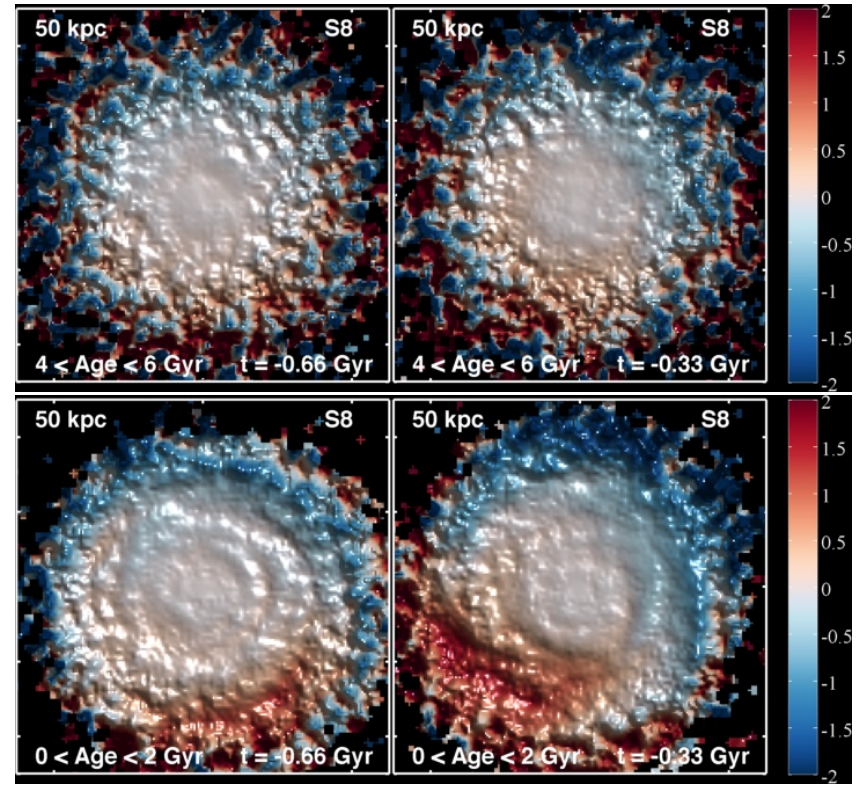

Figure 13. Maps of the mass-weighted mean height, $\langle\mathrm{Z}\rangle$, for the S8 disc at two different times, as indicated in the bottom right corner of each panel. The different colours and the relief indicate different values of $\langle Z\rangle$ in $\mathrm{kpc}$. Top and bottom panels show the $\langle Z\rangle$ maps obtained from stars with ages between of 4 and 6 Gyr, and between 0 and 2 Gyr, respectively.

momentum is well aligned with the semi-minor axis of the dark matter halos within their inner $25 \mathrm{kpc}$, independent of the present-day vertical structure. Interestingly, galaxies that have experienced massive flybys often show DM halos that are well aligned out to distances as large as $80 \mathrm{kpc}$ (S6, S7, S11). This suggests that disc-halo misalignment is not the main driver of their vertical perturbations. In such cases, we find that the satellites do not penetrate far into the host halo. In contrast, galaxies that undergo close encounters with massive companions tend to show significantly misaligned outer DM shells (e.g. S3, S14, S15, S16). Features such as bumps and dips in the evolving orientation of these shells are well correlated with the encounters. Note, however, that although the misaligned outer halo provides a torque on the disc in addition to that from the satellite, the effect due to regions beyond $\sim 50 \mathrm{kpc}$ is negligible in comparison to that from the satellite and the inner halo (see e.g. G16).

A few of our simulations show prominent vertical structures without any recent interaction with a satellite of mass $>M_{\text {min }}$ (e.g. S8 and S13). Other mechanisms, in particular, the accretion of misaligned cold gas (e.g. Sancisi et al. 2008; Aumer \& White 2013; Roškar et al. 2010), are playing a role in these systems. We show that while the vertical patterns are present both in young stars (ages less than 2 Gyr) and in the cold gas in these two galaxies, they are almost absent in the older stars (ages between 4 and 6 Gyr). This contrasts with tidally induced structures, which (in other systems) are almost equally present in stars of all ages. ${ }^{5}$ This is as expected for misaligned accretion either of newly accreted halo gas (e.g. S8) or of cold gas left over after a gas-rich

5 Note however that vertical patterns tend to wash out somewhat faster in kinematically hotter populations (G16).
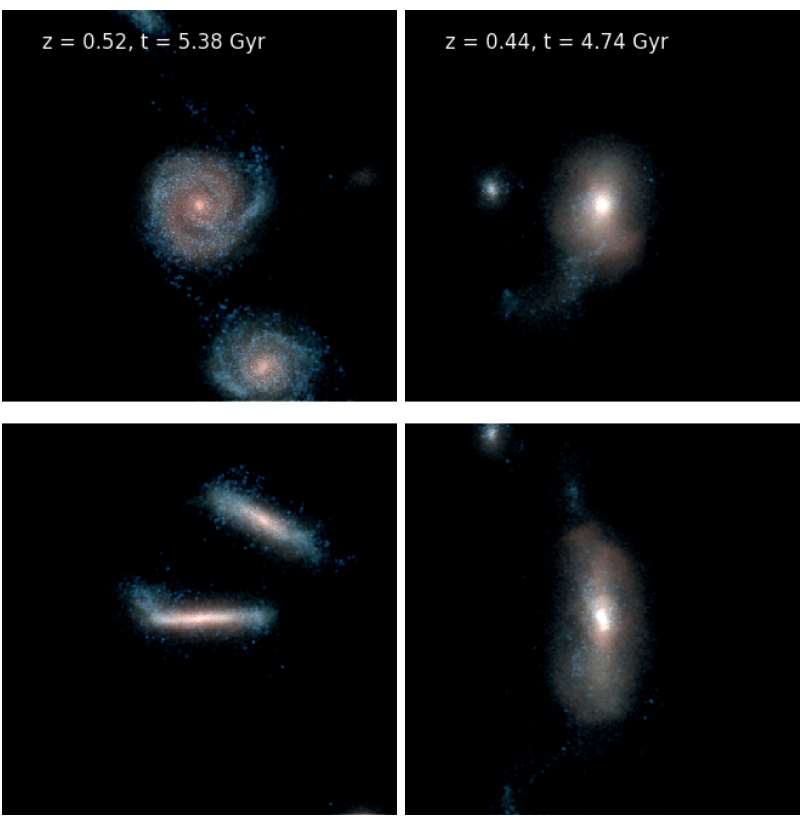

Figure 14. As in Figure 6, but for the galaxy S13. Note that after merging with a massive companion at $t \sim-5$ Gyr the pre-existing discs are completely destroyed.

merger (e.g. S13). In these situations new stars form from the (re)accreted gas and older stars are at most weakly affected.

Several studies have discussed plausible mechanisms to explain the North-South asymmetry observed in the Milky Way's disc; in particular, the Monoceros ring. Some of these papers suggest that this structure could be the result of the tidal disruption of a satellite galaxy on an almost coplanar and nearly circular orbit (e.g. Helmi et al. 2003; Peñarrubia et al. 2005; Michel-Dansac et al. 2011). Interestingly, none of the vertical oscillatory patterns seen in our set of simulations is associated with a tidally disrupted satellite galaxy. Rather, the vertical asymmetries are traced by stars formed in-situ in all cases. Thus, our study suggests that tidal disruption of this kind is less likely as an origin for the North-South asymmetry of the Galactic disc than the other mechanisms we have considered.

\section{ACKNOWLEDGEMENTS}

We would like to thank the referee, James Binney, for an insightful report that led to improvements in the presentation and content of this paper. RG and VS acknowledge support through the DFG Research Centre SFB-881'The Milky Way System' through project A1. VS and RP acknowledges support by the European Research Council under ERC-StG grant EXAGAL-308037.

\section{REFERENCES}

Ann H. B., Park J.-C., 2006, NewA, 11, 293

Aumer M., White S. D. M., 2013, MNRAS, 428, 1055

Aumer M., White S. D. M., Naab T., Scannapieco C., 2013, MNRAS, 434, 3142 
Bailin J., 2003, ApJL, 583, L79

Bailin J. et al., 2005, ApJL, 627, L17

Barrera-Ballesteros J. K. et al., 2014, A\&A, 568, A70

Bershady M. A., Verheijen M. A. W., Swaters R. A., Andersen D. R., Westfall K. B., Martinsson T., 2010, ApJ, 716, 198

Binney J., 1992, ARA\&A, 30, 51

Binney J., Jiang I.-G., Dutta S., 1998, MNRAS, 297, 1237

Binney J., Tremaine S., 2008, Galactic Dynamics: Second Edition. Princeton University Press

Briggs F. H., 1990, ApJ, 352, 15

Cox A. L., Sparke L. S., van Moorsel G., Shaw M., 1996, AJ, 111, 1505

de la Vega A., Quillen A. C., Carlin J. L., Chakrabarti S., D'Onghia E., 2015, MNRAS, 454, 933

Debattista V. P., Sellwood J. A., 1999, ApJL, 513, L107

DeBuhr J., Ma C.-P., White S. D. M., 2012, MNRAS, 426, 983

D'Onghia E., Madau P., Vera-Ciro C., Quillen A., Hernquist L., 2015, ArXiv e-prints

Gómez F. A., Minchev I., O'Shea B. W., Beers T. C., Bullock J. S., Purcell C. W., 2013, MNRAS, 429, 159

Gómez F. A., White S. D. M., Marinacci F., Slater C. T., Grand R. J. J., Springel V., Pakmor R., 2016, MNRAS, 456, 2779

Grand R. J. J. et al., 2016a, ArXiv e-prints

Grand R. J. J., Springel V., Gómez F. A., Marinacci F., Pakmor R., Campbell D. J. R., Jenkins A., 2016b, MNRAS, 459, 199

Helmi A., Navarro J. F., Meza A., Steinmetz M., Eke V. R., 2003, ApJL, 592, L25

Hunter C., Toomre A., 1969, ApJ, 155, 747

Jiang I.-G., Binney J., 1999, MNRAS, 303, L7

Kaiser N. et al., 2010, in Society of Photo-Optical Instrumentation Engineers (SPIE) Conference Series, Vol. 7733, Society of Photo-Optical Instrumentation Engineers (SPIE) Conference Series, p. 0

Kamphuis P., Józsa G. I. G., Oh S.-. H., Spekkens K., Urbancic N., Serra P., Koribalski B. S., Dettmar R.-J., 2015, MNRAS, 452, 3139

Kazantzidis S., Bullock J. S., Zentner A. R., Kravtsov A. V., Moustakas L. A., 2008, ApJ, 688, 254

Kazantzidis S., Zentner A. R., Kravtsov A. V., Bullock J. S., Debattista V. P., 2009, ApJ, 700, 1896

Kim J. H., Peirani S., Kim S., Ann H. B., An S.-H., Yoon S.-J., 2014, ApJ, 789, 90

López-Corredoira M., Cabrera-Lavers A., Garzón F., Hammersley P. L., 2002, A\&A, 394, 883

Marinacci F., Grand R., Pakmor R., Springel V., Gómez F., Frenk C., White S., 2016, ArXiv e-prints

Marinacci F., Pakmor R., Springel V., 2014, MNRAS, 437, 1750

Marinacci F., Vogelsberger M., Mocz P., Pakmor R., 2015, MNRAS, 453, 3999

Martinsson T. P. K., Verheijen M. A. W., Bershady M. A., Westfall K. B., Andersen D. R., Swaters R. A., 2016, A\&A, 585, A99

Michel-Dansac L., Abadi M. G., Navarro J. F., Steinmetz M., 2011, MNRAS, 414, L1

Mihos J. C., 1999, in IAU Symposium, Vol. 186, Galaxy Interactions at Low and High Redshift, Barnes J. E., Sanders D. B., eds., p. 205
Moetazedian R., Just A., 2015, ArXiv e-prints

Momany Y., Zaggia S., Gilmore G., Piotto G., Carraro G., Bedin L. R., de Angeli F., 2006, A\&A, 451, 515

Morganson E. et al., 2016, ArXiv e-prints

Naab T., Ostriker J. P., 2006, MNRAS, 366, 899

Newberg H. J. et al., 2002, ApJ, 569, 245

Ogiya G., Burkert A., 2016, MNRAS, 457, 2164

Ostriker E. C., Binney J. J., 1989, MNRAS, 237, 785

Pakmor R., Springel V., 2013, MNRAS, 432, 176

Pakmor R., Springel V., Bauer A., Mocz P., Munoz D. J., Ohlmann S. T., Schaal K., Zhu C., 2016, MNRAS, 455, 1134

Peñarrubia J. et al., 2005, ApJ, 626, 128

Pranav P., Jog C. J., 2010, MNRAS, 406, 576

Price-Whelan A. M., Johnston K. V., Sheffield A. A., Laporte C. F. P., Sesar B., 2015, MNRAS, 452, 676

Purcell C. W., Bullock J. S., Tollerud E. J., Rocha M., Chakrabarti S., 2011, Nature, 477, 301

Quillen A. C., Minchev I., Bland-Hawthorn J., Haywood M., 2009, MNRAS, 397, 1599

Quinn P. J., Hernquist L., Fullagar D. P., 1993, ApJ, 403, 74

Radburn-Smith D. J. et al., 2014, ApJ, 780, 105

Reshetnikov V., Combes F., 1998, A\&A, 337, 9

Reshetnikov V. P., Mosenkov A. V., Moiseev A. V., Kotov S. S., Savchenko S. S., 2016, MNRAS, 461, 4233

Roškar R., Debattista V. P., Brooks A. M., Quinn T. R., Brook C. B., Governato F., Dalcanton J. J., Wadsley J., 2010, MNRAS, 408, 783

Sancisi R., Fraternali F., Oosterloo T., van der Hulst T., 2008, A\&AR, 15, 189

Schweizer F., Seitzer P., 1992, AJ, 104, 1039

Sellwood J. A., 2013, Dynamics of Disks and Warps, Oswalt T. D., Gilmore G., eds., p. 923

Shen J., Sellwood J. A., 2006, MNRAS, 370, 2

Slater C. T. et al., 2014, ApJ, 791, 9

Sparke L. S., Casertano S., 1988, MNRAS, 234, 873

Springel V., 2005, MNRAS, 364, 1105

Springel V., 2010, MNRAS, 401, 791

Springel V., Hernquist L., 2005, ApJL, 622, L9

Velazquez H., White S. D. M., 1999, MNRAS, 304, 254

Vesperini E., Weinberg M. D., 2000, ApJ, 534, 598

Villalobos Á., Helmi A., 2008, MNRAS, 391, 1806

Vogelsberger M., Genel S., Sijacki D., Torrey P., Springel

V., Hernquist L., 2013, MNRAS, 436, 3031

Weinberg M. D., 1998, MNRAS, 299, 499

Widrow L. M., Barber J., Chequers M. H., Cheng E., 2014, MNRAS, 440, 1971

Widrow L. M., Gardner S., Yanny B., Dodelson S., Chen H.-Y., 2012, ApJL, 750, L41

Xu Y., Newberg H. J., Carlin J. L., Liu C., Deng L., Li J., Schönrich R., Yanny B., 2015, ApJ, 801, 105

Yanny B., Gardner S., 2013, ApJ, 777, 91

Yanny B. et al., 2003, ApJ, 588, 824

York D. G. et al., 2000, AJ, 120, 1579

Younger J. D., Besla G., Cox T. J., Hernquist L., Robertson B., Willman B., 2008, ApJL, 676, L21

Yurin D., Springel V., 2014, ArXiv e-prints 